\title{
Ideal class group annihilators
}

\author{
by
}

\author{
A. Álvarez (Salamanca)
}

A Jesús Muñoz Díaz por su cumpleaños

1. Introduction. The aim of this article is to propose generalizations of Stickelberger's theorem for higher dimensions. Using these results, we study annihilators for some cusp forms of weight 2 . We address certain correspondences, given by sums of Hecke correspondences and defined over Drinfeld modular varieties. This article is motivated by the works of Anderson and Coleman [An1], [C].

Let $\mathbb{P}^{1}$ be the projective line scheme over $\mathbb{F}_{q}, \mathbb{P}^{1} \backslash\{\infty\}=\operatorname{Spec}\left(\mathbb{F}_{q}[t]\right)$ and let $I=p(t) \mathbb{F}_{q}[t]$ be an ideal in $\mathbb{F}_{q}[t]$ with $\operatorname{deg}(p(t))=d+1$. There exists an abelian Galois extension, $K_{I}^{\infty} / \mathbb{F}_{q}(t)$, with group $G_{I} \simeq\left(\mathbb{F}_{q}[t] / I\right)^{\times}$. These fields are Carlitz extensions and are cyclotomic fields in the case of function fields (see $[\mathrm{Ca}]$ ).

Let us consider the $S$-incomplete $L$-function evaluator $(S:=|I| \cup\{\infty\})$

$$
\prod_{x \in \mid \mathbb{P}^{1} \backslash \backslash S}\left(1-\tau_{x} \cdot z^{\operatorname{deg}(x)}\right)^{-1}
$$

$\tau_{x} \in G_{I}$ being the Frobenius element for $x \in\left|\mathbb{P}^{1}\right|$. This Euler product can be expressed as

$$
Q(z)+\frac{\left(\sum_{h \in G_{I}} h\right) \cdot z^{d+1}}{1-q \cdot z},
$$

$Q(z)$ being a polynomial in $\mathbb{Z}\left[G_{I}\right][z]$ of degree $d$. If one writes $Q(z):=$ $\sum_{i=0}^{d} \gamma_{i} \cdot z^{i}$ with $\gamma_{i} \in \mathbb{Z}\left[G_{I}\right]$, then the correspondence

$$
\sum_{i=0}^{d} \Gamma\left(\mathrm{Fr}^{d-i}\right) * \Gamma\left(\gamma_{i}\right)
$$

is trivial on $\operatorname{Spec}\left(K_{I}^{\infty} \otimes K_{I}^{\infty}\right)$. This is proved for $S=\{0,1, \infty\}$ in $[\mathrm{C}]$ and for general $S=\{?, \infty\}$ in [An1]. This result is analogous to the function field

2000 Mathematics Subject Classification: 11G09, 11G20, 11G40, 11F52.

Key words and phrases: Drinfeld modules, Stickelberger's element, cusp forms. 
case of Stickelberger's theorem. Here, $\Gamma\left(\mathrm{Fr}^{i}\right)$ denotes the (transposed) graph of the Frobenius morphism, $\mathrm{Fr}^{i}$, and $\Gamma\left(\gamma_{i}\right)$ is a sum of graphs of elements of $G_{I}$. For arbitrary smooth curves analogous results can be found in [Al2].

These trivial correspondences give an annihilating polynomial for the operator given by the correspondence $\Gamma(\mathrm{Fr})$ acting on the $\mathbb{Q}\left[G_{I}\right]$-module $H^{1}\left(\left(Y_{I}^{\infty}\right)_{\overline{\mathbb{F}}}, \mathbb{Q}_{l}\right)$, and this yields proofs of the Brumer-Stark conjecture in the function field case $([\mathrm{An} 1],[\mathrm{C}],[\mathrm{H} 1],[\mathrm{Ta}],[\mathrm{Al}])^{\prime}, Y_{I}^{\infty}$ denotes the Riemann variety associated with $K_{I}^{\infty} / \mathbb{F}_{q}$.

Here, we study the Euler products

$\prod_{x \in \mid \mathbb{P}^{1} \backslash \backslash S} \frac{1}{1-\sigma_{1}^{x} \cdot z+q \sigma_{2}^{x} \cdot z^{2}-\cdots+(-1)^{n} q^{n(n-1) / 2} \sigma_{n}^{x} \cdot z^{n}}=\sum_{m \geq 0} T(m) \cdot z^{m}$,

where $T(m)$ and $\sigma_{j}^{x}$ are Hecke correspondences over certain modular Drinfeld varieties of dimension $n, \mathcal{E}_{n, ?}^{I \infty}$. For the notation, see Section 2.2.

We prove

THEOREM 1. The correspondence

$$
T(n d)+\Gamma(\mathrm{Fr}) * T(n d-1)+\cdots+\Gamma\left(\operatorname{Fr}^{n d-1}\right) * T(1)+\Gamma\left(\operatorname{Fr}^{n d}\right)
$$

is trivial (= rationally equivalent to 0 as cycles) in $\mathcal{E}_{n, ?}^{I \infty} \times \mathcal{E}_{n, ?}^{I \infty}$.

Here $*$ denotes the product of correspondences. This result for $n=1$ gives us Stickelberger's theorem for cyclotomic function fields, [An1]. To prove it, we study the isogenies of Drinfeld modules, as given in [Gr2].

The schemes $\mathcal{E}_{n, ?}^{I \infty}$ are affine schemes over $\operatorname{Spec}\left(\mathbb{F}_{q}[t, 1 / h(t)]\right)$, and $h(t)$ is a polynomial which depends on $I$. We set $\mathcal{E}_{2}(I \infty):=\mathcal{E}_{2, \text { ? }}^{I \infty} \otimes_{\mathbb{F}_{q}[t]} \mathbb{F}_{q}(t)$. Moreover, $\mathcal{E}(I \infty)$ is a smooth affine curve which is defined over $K_{I}^{\infty}$, and $\overline{\mathcal{E}}(I \infty)$ denotes the associated projective curve over $K_{I}$. Theorem 1 has the following consequence:

Lemma. $T(2 d)+T(2 d-1)+\cdots+T(1)+\Gamma(\mathrm{Id})$ annihilates the group $\operatorname{Pic}(\mathcal{E}(I \infty))$.

It seems to be a Stickelberger theorem for the affine modular curve $\mathcal{E}(I \infty)$ over $K_{I}^{\infty}$.

There exists an arithmetic subgroup, $\Gamma_{I \infty}$, of $G l_{2}\left(\mathbb{F}_{q}[t]\right)$ such that if we denote by $\Omega$ the Drinfeld upper half-plane and by $\bar{M}_{\Gamma_{I \infty}}$ the smooth projective model of the algebraic curve associated with $\Omega / \Gamma_{I \infty}$, then

$$
\bar{M}_{\Gamma_{I \infty}}=\overline{\mathcal{E}}(I \infty) \otimes_{K_{I}^{\infty}} C,
$$

$C$ being the algebraic closure of the completion of $\mathbb{F}_{q}(t)$ at $\infty$. As usual, cusp forms of weight 2 (and type 1 ) for $\Gamma_{I \infty}$ are given by $H^{0}\left(\bar{M}_{\Gamma_{I \infty}}, \Omega_{\bar{M}_{\Gamma_{I \infty}} / C}\right)$. Here we follow the notation and results of [GR]. For the definition and study 
of cusp forms, the readers are referred to the works of Gekeler, Goss or the Habilitationsschrift of Gebhard Böckle.

From the above lemma we obtain an additive version of Stickelberger's theorem for $n=2$ :

TheOREM 2. If the group $\operatorname{Pic}(\mathcal{E}(I \infty))$ is infinite, then there exists a cusp form of weight 2 (and type 1 ) for $\Gamma_{I \infty}$ that is annihilated by $\widetilde{T}(2 d)+\widetilde{T}(2 d-1)$ $+\cdots+\widetilde{T}(1)+$ Id

Here $\widetilde{T}(j)$ is the linear operator given by the $j$-Hecke operator acting on the cusp forms of weight 2 (and type 1).

From Theorem 1, we also obtain ideal class group annihilators for cyclotomic function fields in the spirit of Stickelberger's theorem. We prove that the correspondence

$$
\sum_{i=0}^{n d}\left[\Gamma\left(\operatorname{Fr}^{n d-i}\right) *\left(\sum_{\substack{\operatorname{monic} q(t) \in \mathbb{F}_{q}[t] \\(I, q(t))=1, \operatorname{deg}(q(t))=i}} \varphi(q(t), n) \cdot \Gamma(q(t))\right)\right]
$$

is trivial on $\operatorname{Spec}\left(K_{I}^{\infty} \otimes K_{I}^{\infty}\right)$. Here $\Gamma(q(t))$ denotes the graph of the element of $G_{I}$ associated with the class of $q(t)$ in $\left(\mathbb{F}_{q}[t] / I\right)^{\times}$, and $\varphi(q(t), n)$ is the number of submodules $N \subseteq \mathbb{F}_{q}[t]^{\oplus n}$ such that

$$
\mathbb{F}_{q}[t]^{\oplus n} / N \simeq \mathbb{F}_{q}[t] / q_{1}(t) \oplus \cdots \oplus \mathbb{F}_{q}[t] / q_{n}(t)
$$

with the product of the invariant factors $q_{1}(t) \cdots q_{n}(t)$ equal to $q(t)$. This latter result can also be obtained in a more direct way by using the Euler product of Section 2.4 and Anderson and Coleman's results ([An1], [C]).

Bearing in mind the analogy between Drinfeld varieties in positive characteristic and modular curves for number fields, I believe that the interest of this work is the possible translation of our results to modular curves.

\section{List of notations}

- $\mathbb{F}_{q}$ is a finite field with $q$ elements $\left(q=p^{m}\right)$.

- $\otimes$ denotes $\otimes_{\mathbb{F}_{q}}$.

- $\mathcal{O}_{\mathbb{P}^{1}}$ denotes the ring sheaf of the scheme $\mathbb{P}^{1}$.

- $R$ is an $\mathbb{F}_{q^{-} \text {-algebra. }}$

- $R^{\times}$denotes the group of units in the ring $R$, and Fr the Frobenius morphism.

- $\mathbb{P}_{R}^{1}$ denotes $\mathbb{P}^{1} \otimes R$.

- If $S$ is a finite set of geometric points of $\mathbb{P}^{1}$, then $\mathbb{A}^{S}$ denotes the adele group outside $S$, and $O^{S}$ the adeles within $\mathbb{A}^{S}$ without poles.

- If $M$ is a vector bundle over $\mathbb{P}^{1}$, then $M(k)$ denotes $M \otimes_{\mathcal{O}_{\mathbb{P}} 1} \mathcal{O}_{\mathbb{P}^{1}}(k \infty)$, $k \in \mathbb{Z}$. 
- If $f: X \rightarrow X$ is a morphism of separated schemes, then $\Gamma(f)$ denotes the (transposed) graph of $f, \Gamma(f)=\{(f(x), x): x \in X\}$.

- $\left|\mathbb{P}^{1}\right|$ and $|I|$ denote the geometric points of $\mathbb{P}^{1}$ and $\operatorname{Spec}\left(\mathbb{F}_{q}[t] / I\right)$, respectively.

\section{Elliptic sheaves and Hecke correspondences. Euler products.} In this section, except for Proposition 2.3, all results are valid for any smooth, geometrically irreducible curve over $\mathbb{F}_{q}$ provided with a rational point $\infty$, although we only consider the projective line curve.

2.1. Elliptic sheaves. In this section we recall the definition of elliptic sheaves and level structures over an ideal $I \subset \mathbb{F}_{q}[t]$ ([BlSt], [Dr2], [LRSt], $[\mathrm{Mu}])$.

Definition 2.1. An elliptic sheaf of rank $n$ over $R, E:=\left(E_{j}, i_{j}, \tau\right)$, is a commutative diagram of vector bundles of rank $n$ over $\mathbb{P}_{R}^{1}$, and injective morphisms of modules $\left\{i_{h}\right\}_{h \in \mathbb{N}}, \tau$ :

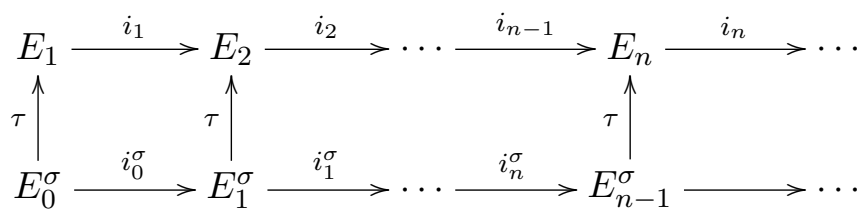

(here $E_{i}^{\sigma}$ denotes $\left.(\operatorname{Id} \times F)^{*} E_{i}\right)$, satisfying:

(a) $\operatorname{deg}\left(\left(E_{j}\right)_{s}\right)=j$ for any $s \in \operatorname{Spec}(R)$.

(b) $E_{j+n}=E_{j}(1)$ for all $j \in \mathbb{Z}$. We can assume that the $i_{k}$ are natural inclusions.

(c) $E_{j}+\tau\left(E_{j}^{\sigma}\right)=E_{j+1}$ for all $j$.

(d) $\alpha^{*}\left(E_{i} / E_{i-1}\right)$ is a rank-one free module over $R, \alpha$ being the natural inclusion $\infty \times \operatorname{Spec}(R) \hookrightarrow \mathbb{P}_{R}^{1}$.

REMARK 1. From these properties, it may be deduced that $h^{0}\left(E_{j}\right)=$ $n+j$ and $h^{1}\left(E_{j}\right)=0, j \geq-n$ ([BlSt], [Dr2]).

Moreover, it is seen that for the $R$-module $H^{0}\left(\mathbb{P}_{R}^{1}, E_{j}\right)(j>-n)$, there exists a basis $\left\{s, \tau s, \ldots, \tau^{n+j-1} s\right\}$ with $\tau s:=\tau\left((\operatorname{Id} \times F)^{*} s\right)$ and $\tau^{h} s:=$ $\tau\left((\mathrm{Id} \times F)^{*} \tau^{h-1} \cdot s\right)$.

Definition 2.2. An I-level structure, $\iota_{I}$, for the elliptic sheaf $\left(E_{j}, i_{j}, \tau\right)$ is an $I$-level structure, $\iota_{j, I}$, for each vector bundle $E_{j}$ compatible with the morphisms $\left\{i_{j}, \tau\right\}$, i.e., $\iota_{j+1, I} \cdot i_{j}=\iota_{j, I}$ and $\iota_{j+1, I} \cdot \tau=(\operatorname{Id} \times F)^{*}\left(\iota_{j, I}\right)$. We denote by $\left(E, \iota_{I}\right)$ an elliptic sheaf with an $I$-level structure.

Recall that an I-level structure for a vector bundle $E_{j}$ over $\mathbb{P}_{R}^{1}$ is a surjective morphism of modules $E_{j} \rightarrow\left(\beta_{*}(R[t] / I)\right)^{\oplus n}$, where $\beta: \operatorname{Spec}(R[t] / I) \hookrightarrow$ $\mathbb{P}_{R}^{1}$ is the natural inclusion. 
The elliptic sheaf $\left(E_{j}, i_{j}, \tau\right)$ defined over $R$ gives a $\tau$-sheaf, $R\{\tau\}=$ $\bigoplus_{i=0}^{\infty} R \cdot \tau^{i}\left(\tau \cdot b=b^{q} \cdot \tau\right)$. One can identify

$$
H^{0}\left(\mathbb{P}_{R}^{1}, E_{j}\right)=\bigoplus_{i=0}^{n+j-1} R \cdot \tau^{i} s,
$$

and in this way $R\{\tau\}$ is isomorphic to the graded $R[t]$-module

$$
\bigcup_{i=0}^{\infty} H^{0}\left(\mathbb{P}_{R}^{1}, E_{j}(i)\right) \text {. }
$$

REMARK 2. By taking the determinant of $\left(E, \iota_{I}\right)$ we obtain an elliptic sheaf of $\operatorname{rank} 1,\left(\operatorname{det}\left(E_{j}\right), \operatorname{det}\left(i_{j}\right), \operatorname{det}(\tau)\right)$, with an $I$-level structure $\operatorname{det}\left(\iota_{I}\right)$. This determinant is studied in detail in $[\mathrm{Ge}]$.

The $\tau$-sheaf associated with $\left(\operatorname{det}\left(E_{j}\right), \operatorname{det}\left(i_{j}\right), \tau_{\text {det }}\right)$ is

$$
R\left\{\tau_{\mathrm{det}}\right\}:=\bigoplus_{i=0}^{\infty} R \cdot \tau_{\mathrm{det}}^{i},
$$

with $\tau_{\text {det }}^{i}=\tau^{i} \wedge \tau^{i+1} \wedge \cdots \wedge \tau^{n+i-1}(i \geq 0)$. Moreover, $\wedge^{n} R\{\tau\}=R\left\{\tau_{\text {det }}\right\}$ as $R[t]$-modules.

We denote $\operatorname{det}\left(E_{j}\right)$ by $L_{j}$, so $\operatorname{deg}\left(L_{j}\right)=j$; recall that $\operatorname{deg}\left(E_{j}\right)=j$.

Proposition 2.3. With the above notations, if $r_{n}-r_{1} \geq n$ then

$$
\tau^{r_{1}} \wedge \tau^{r_{2}} \wedge \cdots \wedge \tau^{r_{n}} \in H^{0}\left(\mathbb{P}_{R}^{1}, L_{r_{n}-n}\right) .
$$

Proof. Since

$\tau_{\text {det }}^{r_{1}}\left(1 \wedge \tau^{r_{2}-r_{1}} \wedge \cdots \wedge \tau^{r_{n}-r_{1}}\right)=\tau^{r_{1}} \wedge \tau^{r_{2}} \wedge \cdots \wedge \tau^{r_{n}} \in H^{0}\left(\mathbb{P}_{R}^{1}, L_{r_{n}-n}\right)$

with $0 \leq r_{1} \leq \cdots \leq r_{n}$, it suffices to prove the result for $r_{1}=0$.

We proceed by induction over $r_{n}$. For $r_{n}=n$, we have to prove that

$$
1 \wedge \tau^{r_{2}} \wedge \cdots \wedge \tau^{n} \in H^{0}\left(\mathbb{P}_{R}^{1}, L_{0}\right) .
$$

Since $t \cdot a_{n}=a_{0}+a_{1} \cdot \tau+\cdots+\tau^{n}$ for some $a_{i} \in R$, there exists $c \in R$ with

$$
1 \wedge \tau^{r_{2}} \wedge \cdots \wedge \tau^{n}=c \cdot\left(1 \wedge \tau^{2} \wedge \cdots \wedge \tau^{n-1}\right) .
$$

Recalling that $0 \leq r_{2} \leq \cdots \leq n$, we conclude that

$$
1 \wedge \tau^{2} \wedge \cdots \wedge \tau^{n-1} \in H^{0}\left(\mathbb{P}_{R}^{1}, L_{0}\right) .
$$

Now assume that the assertion is true for $k<r_{n}$. Set $r_{n}=l+n$. Thus, $1 \wedge \tau^{r_{2}} \wedge \cdots \wedge \tau^{r_{n}}$

$=1 \wedge \cdots \wedge \tau^{r_{n-1}} \wedge\left(t \cdot a_{n} \tau^{r_{n}-n}-a_{0} \tau^{r_{n}-n}+a_{1} \cdot \tau^{r_{n}-n+1}+\cdots+a_{n-1} \tau^{r_{n}-1}\right)$.

Since

$$
1 \wedge \cdots \wedge \tau^{r_{n-1}} \wedge \tau^{r_{n}-i} \in H^{0}\left(\mathbb{P}_{R}^{1}, L_{r_{n}-n}\right) \quad \text { for } i \geq 1
$$


because

$$
1, \ldots, \tau^{r_{n-1}}, \tau^{r_{n}-i} \in H^{0}\left(\mathbb{P}_{R}^{1}, E_{r_{n}-n}\right),
$$

it suffices to prove that

$$
\left(t \cdot a_{n}-a_{0}\right) \cdot\left(1 \wedge \cdots \wedge \tau^{r_{n-1}} \wedge \tau^{r_{n}-n}\right) \in H^{0}\left(\mathbb{P}_{R}^{1}, L_{r_{n}-n}\right) .
$$

Set $k:=\max \left\{r_{n}-n, r_{n-1}\right\}$. If $n \leq k$ then we use the inductive assumption, because $k+1 \leq r_{n}$. When $k \leq n-1$, it suffices to prove that

$$
\left(t \cdot a_{n}-a_{0}\right) \cdot\left(1 \wedge \cdots \wedge \tau^{n-2} \wedge \tau^{n-1}\right) \in H^{0}\left(\mathbb{P}_{R}^{1}, L_{r_{n}-n}\right) .
$$

This is true because $r_{n}-n \geq 1$.

2.2. $\infty$-Level structures. We shall now define level structures at $\infty \in \mathbb{P}^{1}$ over elliptic sheaves of rank 1 . To do so, we take into account the results of [An1, 6.1.1]. We take $t^{-1}$ as a local uniformizer at $\infty$.

The composition of the epimorphism

$$
\mathcal{O}_{\mathbb{P}^{1}}(k) \rightarrow \mathcal{O}_{\mathbb{P}^{1}}(k) / \mathcal{O}_{\mathbb{P}^{1}}(k-1)
$$

with the isomorphism

$$
\mathcal{O}_{\mathbb{P}^{1}}(k) / \mathcal{O}_{\mathbb{P}^{1}}(k-1) \simeq \mathcal{O}_{\mathbb{P}^{1}} / \mathcal{O}_{\mathbb{P}^{1}}(-1)
$$

induced by multiplication by $t^{1-k}$ gives us an $\infty$-level structure over $\mathcal{O}_{\mathbb{P}^{1}}(k)$.

Definition 2.4. We define an $\infty$-level structure for a rank-1 elliptic sheaf, $\left(L_{j}, i_{j}, \tau\right)$, over $R$ to be an $\infty$-level structure $\left(L_{0}, \iota_{\infty}\right)$ such that the diagram

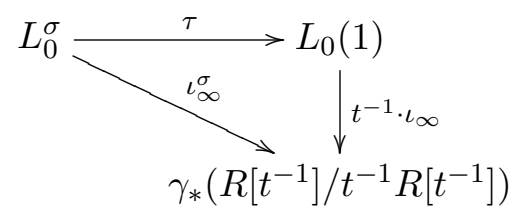

is commutative. Here, $\gamma: \operatorname{Spec}\left(R\left[t^{-1}\right] / t^{-1} R\left[t^{-1}\right]\right) \hookrightarrow \mathbb{P}_{R}^{1}$ is the natural inclusion.

We denote by $\mathcal{E}_{n}^{I}$ and $\mathcal{E}_{n}^{I \infty}$ the moduli of elliptic sheaves with $I$-level structures $\left(E, \iota_{I}\right)$ and with $I+\infty$-level structures, respectively. Here to give an $\infty$-level structure for $E, \iota_{\infty}$, is to give an $\infty$-level structure for the rank-1 elliptic sheaf $\operatorname{det}(E)$. Henceforth, we denote by $\left(E, \iota_{I \infty}\right)$ the element $\left(E, \iota_{I}, \iota_{\infty}\right) \in \mathcal{E}_{n}^{I \infty}$. There exists a morphism, $z: \mathcal{E}_{n}^{I \infty} \rightarrow \operatorname{Spec}\left(\mathbb{F}_{q}[t]\right)$, called the zero morphism, that is defined by

$$
z\left(E, \iota_{I \infty}\right)=\operatorname{supp}\left(E_{0} / \tau\left(E_{-1}^{\sigma}\right)\right)=\operatorname{supp}\left(\operatorname{det}\left(E_{0}\right) / \tau_{\operatorname{det}}\left(\operatorname{det}\left(E_{-1}\right)^{\sigma}\right)\right) .
$$

Bearing in mind the antiequivalence between elliptic sheaves and Drinfeld modules, one can construct a ring $\mathcal{B}_{n}^{I}$ of dimension $n$ such that $\operatorname{Spec}\left(\mathcal{B}_{n}^{I}\right)$ $=\mathcal{E}_{n}^{I}$. For these results see [Dr1], [Dr2], [Lm], [Mu]. 
For $n=1$, it is not hard to obtain a ring $\mathcal{B}_{1}^{I \infty}$ such that

$$
\operatorname{Spec}\left(\mathcal{B}_{1}^{I \infty}\right)=\mathcal{E}_{1}^{I \infty}
$$

Moreover, the morphism of forgetting the $\infty$-level structure

$$
\mathcal{E}_{1}^{I \infty} \rightarrow \mathcal{E}_{1}^{I}
$$

is étale outside $|I|$. In the following remark we calculate $\mathcal{B}_{1}^{I \infty}$ explicitly.

REMARK 3. We consider the rank-1 Drinfeld module $\phi_{t}=a \tau+\bar{t}$, defined over $\mathbb{F}_{q}[\bar{t}, a]$. We shall now study what an $\infty$-level structure for the Drinfeld module $\phi$ is.

Let us consider a rank-1 elliptic sheaf, $\left(L_{j}, i_{j}, \tau\right)$, associated with $\phi$, and let $\iota_{\infty}$ be an $\infty$-level structure for $\left(L_{j}, i_{j}, \tau\right)$. We have the morphisms of modules

$$
\iota_{\infty}: L_{0} \rightarrow \mathbb{F}_{q}[\bar{t}, a]\left[t^{-1}\right] / t^{-1} \mathbb{F}_{q}[\bar{t}, a] .
$$

We choose $s$ with $H^{0}\left(L_{0}\right)=\langle s\rangle$. Note that $s$ is a generator of the line bundle $L_{0}$. We set $\iota_{\infty}(s)=\lambda$, and hence

$$
\iota_{\infty}^{\sigma}: L_{0}^{\sigma} \rightarrow \mathbb{F}_{q}[a, \bar{t}]\left[t^{-1}\right] / t^{-1} \mathbb{F}_{q}[a, \bar{t}]
$$

gives $\iota_{\infty}^{\sigma}(s)=\lambda^{q}$. Also,

$$
t^{-1} \cdot \iota_{\infty}: L_{0}(1) \rightarrow \mathbb{F}_{q}[\bar{t}, a]\left[t^{-1}\right] / t^{-1} \mathbb{F}_{q}[a, \bar{t}]
$$

is such that $t^{-1} \cdot \iota_{\infty}(\tau(s))=t^{-1} \tau s=a^{-1}$, because $t \cdot s=a \cdot \tau s+\bar{t} \cdot s$ and $\bar{t} \cdot t^{-1}=0$ as element in

$$
\mathbb{F}_{q}[a, \bar{t}]\left[t^{-1}\right] / t^{-1} \mathbb{F}_{q}[a, \bar{t}] .
$$

Therefore, the above diagram is commutative if and only if $\lambda^{q}=\lambda \cdot a^{-1}$. Thus, we can choose $\bar{s}:=\lambda \cdot s \in H^{0}\left(M_{0}\right)$ such that $\iota_{\infty}(\bar{s})=1$. Therefore $t \cdot \bar{s}=\tau s+\bar{t}$, and we obtain the Drinfeld module $\bar{\phi}_{t}=\tau+\bar{t}$ isomorphic to $\phi_{t}$. It is not hard to see that $\mathcal{B}_{1}^{\infty}=\mathbb{F}_{q}[\bar{t}]$.

We set $I=p(t) \mathbb{F}_{q}[t]$, where $d+1$ is the degree of $p(t)$. Then

$$
\bar{\phi}_{p(t)}=c_{d+1} \tau^{d+1}+\cdots+c_{1} \tau+p(\bar{t}), \quad c_{i} \in \mathbb{F}_{q}[\bar{t}] .
$$

We have $\mathcal{B}_{1}^{I \infty}=\mathbb{F}_{q}\left[\bar{t}, p(\bar{t})^{-1}, \delta\right]$ with $\delta$ an element of an algebraic closure of $\mathbb{F}_{q}(t)$ satisfying

$$
\phi_{p(t)}(\delta)=\delta^{q^{d+1}}+\cdots+c_{1} \delta^{q}+r(\bar{t}) \delta=0,
$$

and $\phi_{h(t)}(\delta) \neq 0$ with $h(t)$ a proper divisor of $r(t)$. The $I$-level structure for $\left(L_{j}, i_{j}, \tau\right)$ is given by

$$
\iota_{I}(\bar{s})=\bar{\phi}_{t^{r-1}}(\delta)+\bar{\phi}_{t^{d}}(\delta) t+\cdots+\bar{\phi}_{t^{0}}(\delta) t^{d} \in \mathbb{F}_{q}[\bar{t}, \delta][t] / p(t) .
$$

The morphism $\mathbb{F}_{q}[t] \hookrightarrow \mathcal{B}_{1}^{I \infty}(t \mapsto \bar{t})$ gives us the Galois extension $K_{I} / \mathbb{F}_{q}(t)$ with group $\left(\mathbb{F}_{q}[t] / I\right)^{\times}$. 
By considering

$$
\operatorname{det}\left(E, \iota_{I}\right):=\left(\operatorname{det}(E), \operatorname{det}\left(\iota_{I}\right)\right),
$$

and the determinant morphism det $: \mathcal{E}_{n}^{I} \rightarrow \mathcal{E}_{1}^{I}$, we obtain

$$
\mathcal{E}_{n}^{I \infty}=\mathcal{E}_{n}^{I} \times{ }_{\mathcal{E}_{1}^{I}} \mathcal{E}_{1}^{I \infty},
$$

and therefore $\mathcal{E}_{n}^{I \infty}$ is an affine scheme of finite type over $\mathbb{F}_{q}$. It is smooth because the projection $\mathcal{E}_{n}^{I} \times \mathcal{E}_{1}^{I} \mathcal{E}_{1}^{I \infty} \rightarrow \mathcal{E}_{n}^{I}$ is étale since $\mathcal{E}_{1}^{I \infty} \rightarrow \mathcal{E}_{1}^{I}$ is also étale. Note that $\mathcal{E}_{1}^{I \infty}$ is defined over $\mathbb{P}^{1} \backslash(|I| \cup \infty)$.

2.3. Hecke correspondences. We consider $J_{1} \subseteq \cdots \subseteq J_{n}$, a chain of ideals of $\mathbb{F}_{q}[t]$ coprime to $I$, and $S=|I| \cup\{\infty\}$.

Let $\left(E, \iota_{I \infty}\right)$ be an elliptic sheaf defined over $R$ with level structures on $I$ and on $\infty$ and with zero outside $\left|J_{1}\right|$. We denote by $\mathcal{E}_{n,\left|J_{1}\right|}^{I \infty}$ the moduli scheme

$$
\mathcal{E}_{n}^{I \infty} \times_{\mathbb{P}^{1}}\left(\mathbb{P}^{1} \backslash\left|J_{1}\right|\right),
$$

where the fibered product is obtained from the zero morphism $z: \mathcal{E}_{n}^{I \infty} \rightarrow \mathbb{P}^{1}$ and the natural inclusion $\mathbb{P}^{1} \backslash\left|J_{1}\right| \hookrightarrow \mathbb{P}^{1}$.

We denote by

$$
T\left(J_{1}, \ldots, J_{n}\right) \subset \mathcal{E}_{n,\left|J_{1}\right|}^{I \infty} \times \mathcal{E}_{n,\left|J_{1}\right|}^{I \infty}
$$

the Hecke correspondence which is given by the pairs

$$
\left[\left(E, \iota_{I \infty}\right),\left(\bar{E}, \bar{\iota}_{I \infty}\right)\right] \in \mathcal{E}_{n,\left|J_{1}\right|}^{I \infty} \times \mathcal{E}_{n,\left|J_{1}\right|}^{I \infty},
$$

$E$ being a subelliptic sheaf of $\bar{E}$ such that for each $s \in \operatorname{Spec}(R)$ we have

$$
\bar{E}_{s} / E_{s} \simeq k(s)[t] / J_{1} \oplus \cdots \oplus k(s)[t] / J_{n} .
$$

The $I+\infty$-level structure, $\iota_{I \infty}$, defined over $E$ is the composition $\bar{\iota}_{I \infty} \cdot \varrho$, $\varrho$ being the inclusion $E \subset \bar{E}$.

We shall now describe the Hecke correspondences in an adelic way. To do so, consider $\left(\bar{E}, \bar{\iota}_{I \infty}\right)$ defined over an algebraically closed field $K$.

We denote by

$$
\pi_{1}, \pi_{2}: \mathcal{E}_{n,\left|J_{1}\right|}^{I \infty} \times \mathcal{E}_{n,\left|J_{1}\right|}^{I \infty} \rightarrow \mathcal{E}_{n,\left|J_{1}\right|}^{I \infty}
$$

the natural projections. There exists a bijection between the sets:

$$
\pi_{1}\left(\pi_{2}^{-1}\left(\bar{E}, \bar{\iota}_{I \infty}\right) \cap T\left(J_{1}, \ldots, J_{n}\right)\right), \quad \pi_{2}\left(\pi_{1}^{-1}\left(\bar{E}, \bar{\iota}_{I \infty}\right) \cap T\left(J_{1}, \ldots, J_{n}\right)\right),
$$

and $\mathbb{F}_{q}[t]$-modules $M$ and $\bar{M}$,

$$
M \subseteq \mathbb{F}_{q}[t]^{\oplus n} \subseteq \bar{M}
$$

with

$$
\mathbb{F}_{q}[t]^{\oplus n} / M \simeq \bar{M} / \mathbb{F}_{q}[t]^{\oplus n} \simeq \mathbb{F}_{q}[t] / J_{1} \oplus \cdots \oplus \mathbb{F}_{q}[t] / J_{n} .
$$

These sets have the same cardinality, which we denote by $d\left(J_{1}, \ldots, J_{n}\right)$. 
In the following proposition, $\mathcal{C} h t_{n,\left|J_{1}\right|}^{I}$ denotes the stack of shtuckas of rank $n$ with zeroes outside $\left|J_{1}\right|$ and level structures over $I$ (see [Lf]).

Proposition 2.5. The Hecke correspondence $T\left(J_{1}, \ldots, J_{n}\right)$ is a closed subscheme of $\mathcal{E}_{n,\left|J_{1}\right|}^{I \infty} \times \mathcal{E}_{n,\left|J_{1}\right|}^{I \infty}$. Moreover, the morphisms $\pi_{1}, \pi_{2}$ restricted to $T\left(J_{1}, \ldots, J_{n}\right)$ are étale morphisms. We denote these restrictions by $\bar{\pi}_{1}, \bar{\pi}_{2}$, respectively.

Proof. Consider the morphism $\mathfrak{e}: \mathcal{E}_{n,\left|J_{1}\right|}^{I} \rightarrow \mathcal{C} h t_{n,\left|J_{1}\right|}^{I}$ defined by

$$
\mathfrak{e}\left(E, \iota_{I}\right):=\left(\left(E_{-1} \stackrel{i}{\rightarrow} E_{0} \stackrel{\tau}{\leftarrow} E_{-1}^{\sigma}\right), \iota_{I}\right)
$$

(see [Dr3, p. 109]). The Hecke correspondences $\Gamma^{n}(g)$ defined in [Lf, Section I, 4] are closed substacks in $\mathcal{C} h t_{n,\left|J_{1}\right|}^{I} \times \mathcal{C} h t_{n,\left|J_{1}\right|}^{I}$. Let $g \in G l_{n}\left(\mathbb{A}^{S}\right)$ be such that

$$
\bigoplus^{n} O^{S} / g\left(\bigoplus^{n} O^{S}\right) \simeq \mathbb{F}_{q}[t] / J_{1} \oplus \cdots \oplus \mathbb{F}_{q}[t] / J_{n}
$$

as modules. In this way,

$$
T^{I}\left(J_{1}, \ldots, J_{n}\right)=(\mathfrak{e} \times \mathfrak{e})^{*} \Gamma^{n}(g)
$$

is a closed subscheme of $\mathcal{E}_{n}^{I} \times \mathcal{E}_{n}^{I}$, where $T^{I}\left(J_{1}, \ldots, J_{n}\right)$ denotes the Hecke correspondence

$$
\left(\pi_{\infty} \times \pi_{\infty}\right)\left(T\left(J_{1}, \ldots, J_{n}\right)\right) \subset \mathcal{E}_{n,\left|J_{1}\right|}^{I} \times \mathcal{E}_{n,\left|J_{1}\right|}^{I},
$$

$\pi_{\infty}: \mathcal{E}_{n,\left|J_{1}\right|}^{I \infty} \rightarrow \mathcal{E}_{n,\left|J_{1}\right|}^{I}$ being the morphism of forgetting the $\infty$-level structure. Now, $T\left(J_{1}, \ldots, J_{n}\right)$ is the closed subscheme given by the pairs

$$
\left[\left(E, \iota_{I \infty}\right),\left(\bar{E}, \bar{\iota}_{I \infty}\right)\right] \in\left(\pi_{\infty} \times \pi_{\infty}\right)^{-1} T^{I}\left(J_{1}, \ldots, J_{n}\right)
$$

such that

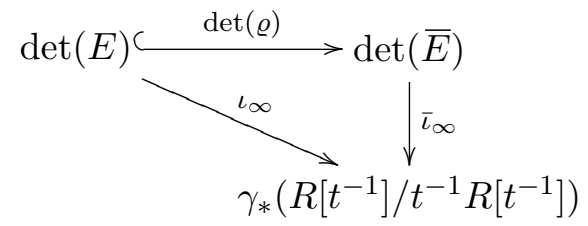

is commutative. Here $\operatorname{det}(\varrho): \operatorname{det}(E) \hookrightarrow \operatorname{det}(\bar{E})$ is the determinant of the injective morphism given between the elliptic sheaves $\varrho: E \hookrightarrow \bar{E}$.

Because

$$
T^{I}\left(J_{1}, \ldots, J_{n}\right)=\Gamma^{n}(g) \times_{\mathcal{C} h t_{n,\left|J_{1}\right|}^{I}} \mathcal{E}_{n,\left|J_{1}\right|}^{I},
$$

and since the projections $p_{i}: \Gamma^{n}(g) \rightarrow \mathcal{C} h t_{n,\left|J_{1}\right|}^{I}(i=1,2)$ are étale morphisms, we see that the two projections from $T^{I}\left(J_{1}, \ldots, J_{n}\right)$ to $\mathcal{E}_{n,\left|J_{1}\right|}^{I}$ are étale morphisms. We conclude that $\bar{\pi}_{1}, \bar{\pi}_{2}$ are étale morphisms because

$$
T\left(J_{1}, \ldots, J_{n}\right)=T^{I}\left(J_{1}, \ldots, J_{n}\right) \times_{\mathcal{E}_{n,\left|J_{1}\right|}^{I}} \mathcal{E}_{n,\left|J_{1}\right|}^{I \infty} .
$$

They are morphisms of degree $d\left(J_{1}, \ldots, J_{n}\right)$. 
The formal sum of Hecke correspondences gives a commutative ring where the product is the composition of correspondences. This ring is isomorphic to the commutative ring

$$
C_{c}\left(K \backslash G l_{n}\left(\mathbb{A}^{S}\right) / K\right)
$$

of $\mathbb{Z}$-valued compactly supported continuous functions on $G l_{n}\left(\mathbb{A}^{S}\right)$, invariant under the action of $K:=G l_{n}\left(O^{S}\right)$ on $G l_{n}\left(\mathbb{A}^{S}\right)$ on the left and on the right. The product is the convolution product. This isomorphism sends the correspondence $T\left(J_{1}, \ldots, J_{n}\right)$ to the characteristic function of the open compact subset

$$
G l_{n}\left(O^{S}\right) \cdot\left(\mu_{J_{1}}, \ldots, \mu_{J_{n}}\right) \cdot G l_{n}\left(O^{S}\right),
$$

with $\mu_{J_{i}} \in \mathbb{A}^{S}$ given by the element $q_{i}(t)$ such that $J_{i}=q_{i}(t) \mathbb{F}_{q}[t]$, and with $\left(\mu_{J_{1}}, \ldots, \mu_{J_{n}}\right)$ denoting the diagonal matrix in $G l_{n}\left(\mathbb{A}^{S}\right)$ with diagonal $\left(\mu_{J_{1}}, \ldots, \mu_{J_{n}}\right)$.

We denote by $T(m)$ the correspondence defined by the formal sum of the Hecke correspondences $T\left(J_{1}, \ldots, J_{n}\right)$, where $\sum_{i=1}^{n} \operatorname{dim}_{\mathbb{F}_{q}} \mathbb{F}_{q}[t] / J_{i}=m$.

As in the number field case, one can consider Hecke correspondences as operators on the abelian group of formal sums of $\mathbb{F}_{q}[t]$-submodules, $N$, of rank $n$ of $\mathbb{F}_{q}(t)^{\oplus n}\left(=\right.$ lattices of $\left.\mathbb{F}_{q}(t)^{\oplus n}\right)$. One defines

$$
T\left(J_{1}, \ldots, J_{n}\right)(N)=\sum \bar{N}
$$

where $\bar{N}$ runs over the submodules of $N$ satisfying:

$$
N / \bar{N} \simeq \mathbb{F}_{q}[t] / J_{1} \oplus \cdots \oplus \mathbb{F}_{q}[t] / J_{n} .
$$

In this way $T(m)(N)=\sum_{\bar{N} \subset N} \bar{N}$, where $\operatorname{dim}_{\mathbb{F}_{q}} N / \bar{N}=m$.

A more rigorous presentation of this section can be found in [Lf] and [Lm].

2.4. Euler products. A generalization to the non-abelian case of the $S$ incomplete $L$-function evaluator at $s=0$ (cf. [H1], [Ta]) is studied in [H2]. In this section we address this issue in another way.

Here, $\mathcal{E}_{n,\left|\mathbb{P}^{1}\right|}^{I \infty}$ denotes the moduli scheme of elliptic sheaves of rank $n$ with level structures over $I$ and $\infty$ and with zero outside $\left|\mathbb{P}^{1}\right|$.

Let $x \in\left|\mathbb{P}^{1}\right| \backslash S(S=|I| \cup\{\infty\})$ and let $t_{x}$ be a local uniformizer for $x$. We consider the diagonal matrix

$$
\left(\mu_{x}, \stackrel{j}{\cdot}, \mu_{x}, 1, \ldots, 1\right) \in G l_{n}\left(\mathbb{A}^{S}\right),
$$

$\mu_{x}$ being the adele in $\mathbb{A}^{S}$ that is 1 over each place of $\left|\mathbb{P}^{1}\right| \backslash S \cup\{x\}$ and $t_{x}$ over $x$. We denote by $\sigma_{j}^{x}, 1 \leq j \leq n$, the Hecke correspondence over $\mathcal{E}_{n,\left|\mathbb{P}^{1}\right|}^{I \infty}$ given by the characteristic function of

$$
G l_{n}\left(O^{S}\right) \cdot\left(\mu_{x}, \stackrel{j}{.}, \mu_{x}, 1, \ldots, 1\right) \cdot G l_{n}\left(O^{S}\right) .
$$


In the following lemma, for ease of notation we assume that $\operatorname{deg}(x)=1$ and $t_{x}$ is a local parameter for $x ; \mathfrak{m}_{x}$ is the maximal ideal associated with $x$.

One can find a proof of the next lemma in [Sh, Th. 3.21]. More or less, we repeat that proof.

LEMma 2.6. We have

$$
\begin{aligned}
& \frac{1}{1-\sigma_{1}^{x} \cdot z+q \sigma_{2}^{x} \cdot z^{2}-q^{3} \sigma_{3}^{x} \cdot z^{3}+\cdots+(-1)^{n} q^{n(n-1) / 2} \sigma_{n}^{x} \cdot z^{n}} \\
&=\sum_{m \geq 0} T^{x}(m) \cdot z^{m}
\end{aligned}
$$

where

$$
T^{x}(m) \subset \mathcal{E}_{n,\left|\mathbb{P}^{1}\right|}^{I \infty} \times \mathcal{E}_{n,\left|\mathbb{P}^{1}\right|}^{I \infty}
$$

denotes the sum of the Hecke correspondences $T\left(\mathfrak{m}_{x}^{r_{1}}, \ldots, \mathfrak{m}_{x}^{r_{n}}\right)$ with $r_{1} \geq$ $\cdots \geq r_{n} \geq 0$ and $r_{1}+\cdots+r_{n}=m$.

Proof. We model this proof after [Ln]. It suffices to prove that for each $r \in \mathbb{N}$ we have "Newton's" formulas

$$
\begin{aligned}
P:= & T^{x}(r)-T^{x}(r-1) \cdot \sigma_{1}^{x}+q \sigma_{2}^{x} \cdot T^{x}(r-2)-\cdots \\
& +(-1)^{n} q^{n(n-1) / 2} T^{x}(r-n) \cdot \sigma_{n}^{x}=0
\end{aligned}
$$

by setting $T^{x}(0)=1$ and $T^{x}(l)=0$ for $l<0$.

To accomplish this, we consider Hecke correspondences as operators over the formal abelian group of lattices, $\bar{N}$ and $N$ being lattices with $\bar{N} \subseteq N$, $\operatorname{dim}_{\mathbb{F}_{q}} N / \bar{N}=r$ and $N / \bar{N}$ concentrated over $x$. We shall prove that the multiplicity of $\bar{N}$ in the formal sum $P(N)$ is 0 .

We have $\sigma_{j}^{x}(N)=\sum N^{\prime}$, where $N^{\prime}$ runs over the sublattices of $N$ with

$$
N / N^{\prime} \simeq \mathbb{F}_{q}[t] / \mathfrak{m}_{x} \oplus \stackrel{j}{\stackrel{j}{*}} \oplus \mathbb{F}_{q}[t] / \mathfrak{m}_{x}
$$

or, equivalently, the vector subspaces of codimension $j$ of $N / \mathfrak{m}_{x} \cdot N$.

Set $h:=\operatorname{dim}_{\mathbb{F}_{q}} N /\left(\mathfrak{m}_{x} \cdot N+\bar{N}\right)$. The number of lattices $N^{\prime}$ such that $\bar{N} \subset N^{\prime}$ is given by the number of $\mathbb{F}_{q}$-vector subspaces in $N /\left(\mathfrak{m}_{x} \cdot N+\bar{N}\right)$ of codimension $j$. This number is given by the $q$-combinatorial number

$$
\left(\begin{array}{l}
h \\
j
\end{array}\right)_{q}:=\frac{\left(q^{h}-1\right) \cdots\left(q^{h-j+1}-1\right)}{\left(q^{j}-1\right) \cdots(q-1)}
$$

for $j \leq h$, and $\left(\begin{array}{l}h \\ j\end{array}\right)_{q}:=0$ for either $j<0$ or $j>h$.

We conclude the proof bearing in mind the relation

$$
\left(\begin{array}{l}
h \\
h
\end{array}\right)_{q}-\left(\begin{array}{c}
h \\
h-1
\end{array}\right)_{q}+q \cdot\left(\begin{array}{c}
h \\
h-2
\end{array}\right)_{q}-\cdots+(-1)^{n} q^{n(n-1) / 2} \cdot\left(\begin{array}{c}
h \\
h-n
\end{array}\right)_{q}=0 .
$$

I have taken this formula from [Lm, Appendix D] (cf. [Ma]). 
Theorem 2.7. Set

$$
L^{x}:=\frac{1}{1-\sigma_{1}^{x} \cdot z+q \sigma_{2}^{x} \cdot z^{2}-q^{3} \sigma_{3}^{x} \cdot z^{3}+\cdots+(-1)^{n} q^{n(n-1) / 2} \sigma_{n}^{x} \cdot z^{n}} .
$$

Then

$$
\prod_{x \in \mid \mathbb{P}^{1} \backslash \backslash S} L^{x}=\sum_{m \geq 0} T(m) \cdot z^{m} .
$$

Proof. It suffices apply the above lemma bearing in mind that if $J_{1}$ and $\bar{J}_{1}$ are coprime ideals in $\mathbb{F}_{q}[t]$, then

$$
T\left(J_{1}, \ldots, J_{n}\right) \cdot T\left(\bar{J}_{1}, \ldots, \bar{J}_{n}\right)=T\left(J_{1} \cdot \bar{J}_{1}, \ldots, J_{n} \cdot \bar{J}_{n}\right) .
$$

3. Isogenies and Hecke correspondences. Here, we study the isogenies between Drinfeld modules (= elliptic sheaves) [Gr2] to establish the relation between these isogenies and the above Euler products.

3.1. Isogenies for elliptic sheaves

Definition 3.1. An isogeny, $\Phi$, of degree $m \in \mathbb{N}$ between two elliptic sheaves with $I$-level structures $\left(E, \iota_{I \infty}\right),\left(\bar{E}, \bar{\iota}_{I \infty}\right)$ and $\infty$-level structures for $\operatorname{det}(E)$ and $\operatorname{det}(\bar{E})$ is a morphism of modules $\Phi_{i}: E_{i} \rightarrow \bar{E}_{i+m}$, for each $i$, with $\operatorname{Im}\left(\Phi_{i}\right) \not \subset \bar{E}_{i+m-1}$, preserving the diagrams that define the elliptic sheaves and their level structures.

If $E$ and $\bar{E}$ are defined over $R$, then to give an isogeny $\Phi: E \rightarrow \bar{E}$ of degree $m$ is equivalent to giving a morphism of $\tau$-sheaves $\phi: R\{\tau\} \rightarrow$ $R\{\bar{\tau}\}$ such that if $r(\tau)$ is a monic polynomial with $\operatorname{deg}_{\tau}(r(\tau))=j$ then $\operatorname{deg}_{\bar{\tau}} \phi(r(\tau))=m+j$.

Lemma 3.2. Let $M$ and $N$ be vector bundles of rank $n$ over $\mathbb{P}_{R}^{1}$, and with $x$ a rational point of $\mathbb{P}^{1}$. If $f: M \rightarrow N$ is a morphism of modules such that its restriction to $k(x)$

$$
f_{\mid k(x) \otimes R}: M_{\mid k(x) \otimes R} \rightarrow N_{\mid k(x) \otimes R}
$$

is an isomorphism, then $f$ is injective.

Proof. Assume that $x$ is the rational point $0 \in \mathbb{P}^{1}$. We have the exact sequence

$$
0 \rightarrow K \rightarrow M \stackrel{f}{\rightarrow} N
$$

If we prove that $K_{\left(\mathbb{P}_{1} \backslash\{\infty\}\right) \otimes R}=0$ then we conclude the proof. Let

$$
0 \rightarrow \widehat{K} \rightarrow \widehat{M} \stackrel{\widehat{f}}{\rightarrow} \widehat{N}
$$


be the completion of the above exact sequence along the ideal $t R[t]$. By hypothesis, $f_{\mid k(x) \otimes R}$ is an isomorphism. One deduces that $\widehat{f}$ is also an isomorphism and hence $\widehat{K}=0$, since

$$
\operatorname{Spec}_{\text {maximal }}(R[[t]])=0 \times \operatorname{Spec}_{\text {maximal }}(R),
$$

and in view of the Nakayama lemma. If we prove that the natural morphism $g: K \rightarrow \widehat{K}$ is injective we are done. By the Krull theorem, if $g(a)=0$ then there exists $1+t \cdot q(t) \in R[t]$ such that $(1+t \cdot q(t)) \cdot a=0$. However, the homothety morphism given by $1+t \cdot q(t)$ over $R[t]$ is injective and therefore it is also injective over $M$, because $M$ is locally free. Hence, $a=0$.

LEMMA 3.3. In the above notation, if $\Phi$ is an isogeny of degree $m \leq n d$ between $\left(E, \iota_{I \infty}\right),\left(\bar{E}, \bar{\iota}_{I \infty}\right)$ then $\Phi$ is injective, and it is the only isogeny between these elliptic sheaves with level structures. Moreover, there exists a maximal $r \in \mathbb{N}(r \leq n d)$ such that $\phi(R\{\tau\}) \subseteq R\{\bar{\tau}\} \cdot \bar{\tau}^{r}$.

Proof. We can assume that the elliptic sheaves are defined over an $\mathbb{F}_{q^{-}}$ algebra $R$. In this way, the injectivity is deduced from the above lemma. We denote by $I$ indiscriminately the ideal in $\mathbb{F}_{q}[t]$ and the ideal sheaf in $\mathcal{O}_{\mathbb{P}^{1}}$.

Let $\Phi^{\prime}$ be another isogeny; $\Phi-\Phi^{\prime}$ defines a morphism $E_{0} \rightarrow I \cdot \bar{E}_{n d}$. Since $E_{0}$ is generated by its global sections, and since $\operatorname{deg}\left(I \cdot \bar{E}_{n d}\right)=-n$, because $\operatorname{deg}(I)=d+1$, we have $h^{0}\left(I \cdot \bar{E}_{n d}\right)=0$. Hence $\Phi=\Phi^{\prime}$.

The last assertion of the lemma is evident.

We consider $\left|\mathbb{P}_{1}\right|_{n d}$, the subset of geometric points of $\mathbb{P}_{1}$ of degree less than or equal to $n d$. Let $\mathcal{E}_{n,\left|\mathbb{P}^{1}\right|_{n d}}$ denote the moduli scheme of elliptic sheaves of rank $n$ with level structures over $I$ and $\infty$ and with zero outside $\left|\mathbb{P}^{1}\right|_{n d}$.

LEMMA 3.4. With the above notations, the set

$$
\left[\left(E, \iota_{I \infty}\right),\left(\bar{E}, \bar{\iota}_{I \infty}\right)\right] \in \mathcal{E}_{n,\left|\mathbb{P}_{1}\right|_{n d}}^{I \infty} \times \mathcal{E}_{n,\left|\mathbb{P}_{1}\right|_{n d}}^{I \infty}
$$

such that there exists an isogeny of degree $m \leq n d$ between $\left(E, \iota_{I \infty}\right)$ and $\left(\bar{E}, \bar{\iota}_{I \infty}\right)$ with $r=0$ is given by the correspondence $T(m) \subset \mathcal{E}_{n,\left|\mathbb{P}_{1}\right|_{n d}}^{I \infty} \times$ $\mathcal{E}_{n,\left|\mathbb{P}_{1}\right|_{n d}}^{I \infty}$

Proof. It is clear that a pair in $T(m)$ defines an isogeny of degree $m$ with the required properties. Moreover, Lemma 3.3 asserts that there only exists one isogeny of degree $m \leq n d$ between two elliptic sheaves with $I$-level structures. With this result, one deduces that if $\left(E, \iota_{I \infty}\right)$ and $\left(E^{\prime}, \iota_{I \infty}^{\prime}\right)$ are subelliptic sheaves with level structures of $\left(\bar{E}, \bar{\iota}_{I \infty}\right)$ by two different isogenies of degree $m$, then $\left(E, \iota_{I \infty}\right)$ is not isomorphic to $\left(E^{\prime}, \iota_{I \infty}^{\prime}\right)$.

On the other hand, if $\Phi:\left(E, \iota_{I \infty}\right) \rightarrow\left(\bar{E}, \bar{\iota}_{I \infty}\right)$ is an isogeny with $r=0$ and degree $m$, then by the serpent lemma we have isomorphisms $(\operatorname{Id} \times F)^{*}\left(\bar{E}_{i+m} / \Phi_{i}\left(E_{i}\right)\right) \simeq \bar{E}_{i+m} / \Phi_{i}\left(E_{i}\right)$ for each integer $i$. Since the zeroes 
of the elliptic sheaves considered are of degree $>n d$, we have

$$
\bar{E}_{i+m} / \Phi_{i}\left(E_{i}\right) \simeq R[t] / J_{1} \oplus \cdots \oplus R[t] / J_{n},
$$

where $J_{1} \subseteq \cdots \subseteq J_{n}$ are ideals in $\mathbb{F}_{q}[t]$ coprime to $I$ with $\sum_{i=0}^{n} \operatorname{dim}_{\mathbb{F}_{q}} A / J_{i}=m$. Here, we have assumed that $\left(E, \iota_{I \infty}\right)$ and $\left(\bar{E}, \bar{\iota}_{I \infty}\right)$ are defined over $R$.

COROLlary 3.5. The subset of pairs $(e, \bar{e}) \in \mathcal{E}_{n,\left|\mathbb{P}_{1}\right|_{n d}}^{I \infty} \times \mathcal{E}_{n,\left|\mathbb{P}_{1}\right|_{n d}}^{I \infty}$ such that there exists an isogeny of degree nd is given by the correspondence

$$
T(n d)+\Gamma(\mathrm{Fr}) * T(n d-1)+\cdots+\Gamma\left(\mathrm{Fr}^{n d-1}\right) * T(1)+\Gamma\left(\mathrm{Fr}^{n d}\right) .
$$

Here, $\Gamma\left(\mathrm{Fr}^{i}\right)$ is given by the graph of the $q^{i}$-Frobenius morphism, and * denotes the product of correspondences.

Proof. The elliptic sheaf associated with the $\tau$-sheaf $R\{\bar{\tau}\} \cdot \bar{\tau}^{r}$ is

$$
\left[(\mathrm{Id} \times \mathrm{Fr})^{r}\right]^{*} \bar{E} \text {. }
$$

In view of the two last lemmas, the corollary is deduced from the fact that between $E_{0}$ and $\left[(\mathrm{Id} \times \mathrm{Fr})^{n d+j}\right]^{*} \bar{E}_{-j}$ there is no injective morphism for $j>0$, because $\operatorname{deg}\left(E_{0}\right)=0$ and $\operatorname{deg}\left[\left((\operatorname{Id} \times \operatorname{Fr})^{n d+j}\right)^{*} \bar{E}_{-j}\right]=-j$.

3.2. Trivial correspondences. In this section we shall prove that the correspondence of the above Corollary 3.5 is trivial.

Proposition 3.6. Let $M$ be a vector bundle over $\mathbb{P}_{R}^{1}$ of rank $n$ and degree 0 where $h^{0}(M(-1))=h^{1}(M(-1))=0$, and with an I-level structure $\iota_{I}$. Then $H^{0}\left(\mathbb{P}_{R}^{1}, M\right)$ is a free $R$-module of rank $n$, and $M \simeq H^{0}\left(\mathbb{P}_{R}^{1}, M\right) \otimes \mathcal{O}_{\mathbb{P}_{1}}$.

Proof. If $x \in \operatorname{Spec}\left(\mathbb{F}_{q}[t] / I\right)$ is a rational point, then $h^{0}(M(-x))=$ $h^{1}(M(-x))=0$. Bearing in mind the morphism given by the $x$-level structure $\iota_{x}: M \rightarrow(k(x) \otimes R)^{n}$, we obtain an isomorphism

$$
M / M(-x) \simeq\left(R[t] / \mathfrak{m}_{x}\right)^{\oplus n} .
$$

Therefore, by taking global sections in the exact sequence of $\mathcal{O}_{\mathbb{P}_{1}} \otimes R$-modules

$$
0 \rightarrow M(-x) \rightarrow M \rightarrow M / M(-x) \rightarrow 0
$$

we conclude the proof.

The argument is valid when $\operatorname{Spec}\left(\mathbb{F}_{q}[t] / I\right)$ does not have rational points, because $N$ is an $R$-free module if and only if $N \otimes \mathbb{F}_{q^{d}}$ is an $R \otimes \mathbb{F}_{q^{d}}$-free module.

If $\left(\bar{M}, \bar{\iota}_{I}\right)$ is an $I$-level structure then we denote by $\left(\bar{M}(d), \bar{\iota}_{I}(d)\right)$ the $I$-level structure over $\bar{M}(d)$ obtained from $\bar{\iota}_{I}$ by considering the natural inclusion $\bar{M} \subseteq \bar{M}(d)$. Recall that $\infty \notin|I|$.

Lemma 3.7. If $\left(\bar{M}, \bar{\iota}_{I}\right),\left(M, \iota_{I}\right)$ are level structures over $R$, where $M$ and $\bar{M}$ satisfy the conditions of the above proposition, then there exists a morphism of vector bundles, $h: M \rightarrow \bar{M}(d)$, such that the diagram 


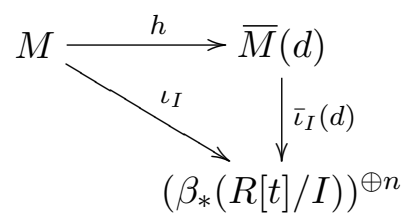

is commutative.

Here $h$ is said to be a morphism between $\left(M, \iota_{I}\right)$ and $\left(\bar{M}(d), \bar{\iota}_{I}(d)\right)$.

Proof. If we choose a base $\left\{s_{1}, \ldots, s_{n}\right\}$ for $H^{0}\left(\mathbb{P}_{R}^{1}, M\right)$, then $H^{0}\left(\iota_{I}\right)$ : $H^{0}(M) \rightarrow(R[t] / I)^{\oplus n}$ has the associated matrix

$$
\Delta_{0}+\Delta_{1} t+\cdots+\Delta_{d} t^{d},
$$

where $\Delta_{i}$ are $n \times n$-matrices with entries in $R$.

We have

$$
H^{0}\left(\mathbb{P}_{R}^{1}, \bar{M}(d)\right)=\bigoplus_{i=0}^{d} H^{0}\left(\mathbb{P}_{R}^{1}, \bar{M}\right) \cdot t^{i} .
$$

Moreover, bearing in mind that $\operatorname{deg}(I)=d+1$ we also have

$$
H^{0}\left(\mathbb{P}_{R}^{1}, \bar{M}(d)\right) \stackrel{H^{0}\left(\bar{\iota}_{I}\right)}{\simeq}(R[t] / I)^{\oplus n},
$$

because $h^{0}(I \cdot \bar{M}(d))=0$. Thus, $H^{0}(h)$ must satisfy

$$
H^{0}(h)=A_{0}+\cdots+A_{d} \cdot t^{d}:=\left(H^{0}\left(\bar{\iota}_{I}\right)\right)^{-1} \cdot\left(\Delta_{0}+\Delta_{1} t+\cdots+\Delta_{d} t^{d}\right),
$$

where $A_{i}$ are $n \times n$-matrices with entries in $R$. We conclude the proof by invoking the above proposition.

The same arguments of Lemma 3.3 allow us to deduce that $h$ is unique.

By Remark 1, if $E$ is an elliptic sheaf of rank $n$, then $E_{0}$ satisfies the conditions of Proposition 3.6.

Let us consider the elliptic sheaves, defined over $R$, with $I$-level structures $\left(E, \iota_{I \infty}\right),\left(\bar{E}, \bar{\iota}_{I \infty}\right)$ and $\infty$-level structures for $\operatorname{det}(E)$ and $\operatorname{det}(\bar{E})$.

Lemma 3.8. Let $h:\left(E_{0}, \iota_{0, I}\right) \rightarrow\left(\bar{E}_{0}(d), \bar{\iota}_{0, I}(d)\right)$ be the morphism between vector bundles with level structures given in Lemma 3.7, and let $\iota_{\infty}$, $\bar{\iota}_{\infty}$ be level structures at $\infty$ over $\operatorname{det}(E)$ and $\operatorname{det}(\bar{E})$, respectively. Then there exists an isogeny $\Phi:\left(E, \iota_{I \infty}\right) \rightarrow\left(\bar{E}, \bar{\iota}_{I \infty}\right)$ of degree nd with $\Phi_{0}=h$ if and only if $\operatorname{det}(h)$ is a morphism for the level structures $\left(\operatorname{det}\left(E_{0}\right), \iota_{\infty}\right)$, $\left(\operatorname{det}\left(\bar{E}_{0}(d)\right), \bar{\iota}_{\infty}\right)\left(\right.$ i.e. $\left.\bar{\iota}_{\infty} \cdot \operatorname{det}(h)=\iota_{\infty}\right)$, and the morphism of $R[t]$-modules given by $h, h_{A}: R\{\tau\} \rightarrow R\{\bar{\tau}\}$, satisfies

$$
\operatorname{deg}_{\bar{\tau}}\left(h_{A}(1)\right) \leq n-2+n d, \ldots, \operatorname{deg}_{\bar{\tau}}\left(h_{A}\left(\tau^{n-2}\right)\right) \leq n-2+n d .
$$

Proof. The direct implication is trivial. 
We prove the converse. Since $h$ is a morphism for the $I$-level structures $\left(E_{0}, \iota_{0, I}\right)$ and $\left(\bar{E}_{0}(d), \bar{\iota}_{0, I}(d)\right)$, we have

$$
h_{A}(\tau)-\bar{\tau} \cdot h_{A}(1), \ldots, h_{A}\left(\tau^{n-1}\right)-\bar{\tau} \cdot h_{A}\left(\tau^{n-2}\right) \in I \cdot R\{\bar{\tau}\} .
$$

Moreover, since $\operatorname{deg}(I)=d+1$, if $r(\bar{\tau}) \neq 0 \in R\{\bar{\tau}\} \cdot I$ then $\operatorname{deg}_{\bar{\tau}}(r(\bar{\tau})) \geq$ $n(d+1)$. Thus, by the hypothesis of the lemma we deduce that

$$
h_{A}(\tau)-\bar{\tau} \cdot h_{A}(1)=\cdots=h_{A}\left(\tau^{n-1}\right)-\bar{\tau} \cdot h_{A}\left(\tau^{n-2}\right)=0 .
$$

Therefore, $h_{A}\left(\tau^{i}\right)=\bar{\tau}^{j} \cdot h_{A}\left(\tau^{k}\right)$ for $j+k=i$ and $1 \leq i \leq n-1$.

Now, we prove the equalities

$$
\begin{aligned}
\operatorname{deg}_{\bar{\tau}}\left(h_{A}(1)\right)=n d, \quad \operatorname{deg}_{\bar{\tau}}\left(h_{A}(\tau)\right)= & n d+1, \ldots, \\
& \operatorname{deg}_{\bar{\tau}}\left(h_{A}\left(\tau^{n-1}\right)\right)=n d+n-1 .
\end{aligned}
$$

We consider the determinant elliptic sheaves $\operatorname{det}(E)$ and $\operatorname{det}(\bar{E})$ and their $\tau$-sheaves $R\left\{\tau_{\text {det }}\right\}$ and $R\left\{\bar{\tau}_{\text {det }}\right\}$, respectively. We see that

$$
\left[\operatorname{det}\left(h_{A}\right) \cdot \tau_{\text {det }}-\bar{\tau}_{\text {det }} \cdot \operatorname{det}\left(h_{A}\right)\right]\left(1 \wedge \tau \wedge \cdots \wedge \tau^{n-2} \wedge \tau^{n-1}\right)
$$

is an element of $R\left\{\tau_{\text {det }}\right\}$ of degree $\leq n d+1$. However, by hypothesis $\operatorname{det}(h)$ is a morphism for $\infty$-level structures for elliptic sheaves and therefore this element is of degree $\leq n d$.

Because $h_{A}\left(\tau^{i}\right)=\bar{\tau} \cdot h_{A}\left(\tau^{i-1}\right)$ for $1 \leq i \leq n-1$, the above element of $R\left\{\tau_{\text {det }}\right\}$ is equal to

$$
h_{A}(\tau) \wedge \cdots \wedge h_{A}\left(\tau^{n-1}\right) \wedge\left[h_{A} \cdot \tau-\bar{\tau} \cdot h_{A}\right]\left(\tau^{n-1}\right) .
$$

Since $\operatorname{deg}_{\bar{\tau}}\left(h_{A}\left(\tau^{n-1}\right)\right) \leq n d+n-1$ and $h_{A}\left(\tau^{i}\right)=\bar{\tau}^{i} \cdot h_{A}(1)$ for $1 \leq i \leq$ $n-1$, we have the inequalities

$\operatorname{deg}_{\bar{\tau}}\left(h_{A}(1)\right) \leq n d, \operatorname{deg}_{\bar{\tau}}\left(h_{A}(\tau)\right) \leq n d+1, \ldots, \operatorname{deg}_{\bar{\tau}}\left(h_{A}\left(\tau^{n-1}\right)\right) \leq n d+n-1$. But $\bar{E}_{0}(d) / h\left(E_{0}\right)$ is not concentrated at $\infty$, because $\bar{\iota}_{\infty} \cdot \operatorname{det}(h)=\iota_{\infty}$ and $\bar{\iota}_{\infty}, \iota_{\infty}$ are surjective morphisms, and hence the equalities $(*)$ follow.

Using Remark 2, since

$$
h_{A}(\tau) \wedge \cdots \wedge h_{A}\left(\tau^{n-1}\right) \wedge\left[h_{A} \cdot \tau-\bar{\tau} \cdot h_{A}\right]\left(\tau^{n-1}\right)
$$

is an element of $R\left\{\tau_{\text {det }}\right\}$ of degree $\leq n d$, we have

$$
\operatorname{deg}_{\bar{\tau}}\left[h_{A} \cdot \tau-\bar{\tau} \cdot h_{A}\right]\left(\tau^{n-1}\right) \leq n d+n-1,
$$

and we conclude that $\left[h_{A} \cdot \tau-\bar{\tau} \cdot h_{A}\right]\left(\tau^{n-1}\right)=0$, because

$$
\left[h_{A} \cdot \tau-\bar{\tau} \cdot h_{A}\right]\left(\tau^{n-1}\right) \in R\{\bar{\tau}\} \cdot I
$$

and $\operatorname{deg}(I)=d+1$. Thus, $h_{A}: R\{\tau\} \rightarrow R\{\bar{\tau}\}$ is an isogeny of degree $n d$.

Lemma 3.9. Let $X=\operatorname{Spec}(A)$ be a smooth, noetherian scheme of dimension $2 n$. Let $Z_{1}+\cdots+Z_{r}$ be an $n$-cycle in $X$ such that the $Z_{i}$ are different irreducible closed subschemes of dimension $n$ in $X$. If the closed 
subscheme $Z:=Z_{1} \cup \cdots \cup Z_{r}$ is given by an ideal generated by $n$ elements $a_{1}, \ldots, a_{n} \in A$, then the $n$-cycle $Z_{1}+\cdots+Z_{r}$ is rationally equivalent to 0 .

Proof. Let $\mathfrak{I}$ be an ideal in $A$. We denote by $Z(\mathfrak{I})$ the cycle associated in $X$ with the closed subscheme given by $\mathfrak{I}$. The prime ideal in $A$ given by $Z_{i}$ is denoted by $P_{i}$. Thus,

$$
Z\left(P_{1} \cap \cdots \cap P_{r}\right)=Z_{1}+\cdots+Z_{r} .
$$

Consider the ideal $\left(a_{2}, \ldots, a_{n}\right)$ in $A$ generated by $a_{2}, \ldots, a_{n}$ and let $Q_{1} \cap$ $\ldots \cap Q_{h}$ be a minimal primary decomposition of this ideal. If $Y_{1}, \ldots, Y_{k}$ $(k \leq h)$ are the irreducible components of the closed subscheme in $X$ given by $\left(a_{2}, \ldots, a_{n}\right)$, then $\operatorname{dim} Y_{j} \geq n+1$. We may assume, reordering the indices, that $Z\left(Q_{1}\right)=Y_{1}, \ldots, Z\left(Q_{k}\right)=Y_{k}$.

By taking the localization with respect to $P_{i}$, one obtains

$$
\left(A / Q_{1} \cap \cdots \cap Q_{h}\right)_{P_{i}},
$$

which is a local ring of dimension 1 because $\operatorname{dim} Y_{j} \geq n+1$ for all $j$. From the equality of rings

$$
A / Q_{1} \cap \cdots \cap Q_{h}+\left(a_{1}\right)=A / P_{1} \cap \cdots \cap P_{r}
$$

one obtains

$$
\left(A / Q_{1} \cap \cdots \cap Q_{h}+\left(a_{1}\right)\right)_{P_{i}}=\left(A / P_{i}\right)_{P_{i}} .
$$

Therefore, $\left(A / Q_{1} \cap \cdots \cap Q_{h}\right)_{P_{i}}$ is principal and hence an integral domain, and therefore there exists a unique $Q_{l_{i}}\left(l_{i} \leq k\right)$ with $Q_{l_{i}} \subset P_{i}$. If we denote by $P_{j_{1}}, \ldots, P_{j_{m_{j}}}$ the $P_{i}$ 's with $Q_{j} \subset P_{j_{1}}, \ldots, Q_{j} \subset P_{j_{m_{j}}}(j \leq k)$, then within the $n+1$-dimensional scheme $Z\left(Q_{j}\right)=Y_{j}$,

$$
Z_{j_{1}}+\cdots+Z_{j_{m_{j}}}
$$

is given by the zero locus of $a_{1}$, which proves that $Z_{j_{1}}+\cdots+Z_{j_{m_{j}}}$ is rationally equivalent to 0 on $X$. This yields the conclusion, because $Z_{1}+\cdots+Z_{r}=$ $\sum_{j=1}^{k}\left(Z_{j_{1}}+\cdots+Z_{j_{m_{j}}}\right)$.

THEOREM 3.10. The correspondence

$$
T_{I}^{n}:=T(n d)+\Gamma(\mathrm{Fr}) * T(n d-1)+\cdots+\Gamma\left(\mathrm{Fr}^{n d-1}\right) * T(1)+\Gamma\left(\mathrm{Fr}^{n d}\right)
$$

is trivial (= rationally equivalent to 0 as an n-cycle in $\mathcal{E}_{n,\left|\mathbb{P}_{1}\right|_{n d}}^{I \infty} \times \mathcal{E}_{n,\left|\mathbb{P}_{1}\right|_{n d}}^{I \infty}$ ).

Proof. In view of Corollary 3.5 and Lemma 3.8, this correspondence is given by the zero locus in $n$ regular functions in $\mathcal{E}_{n,\left|\mathbb{P}_{1}\right|_{n d}}^{I \infty} \times \mathcal{E}_{n,\left|\mathbb{P}_{1}\right|_{n d}}^{I \infty}$.

We are under the hypotheses of the above lemma because the projection on the first entry, $T(r) \rightarrow \mathcal{E}_{n,\left|\mathbb{P}_{1}\right|_{n d}}^{I \infty}$, is an étale morphism and therefore $T(r)$ is smooth. Moreover, by Lemma 3.3, if $i, j \leq n d$ and $i \neq j$ then

$$
\Gamma\left(\mathrm{Fr}^{i}\right) * T(n d-i) \cap \Gamma\left(\mathrm{Fr}^{j}\right) * T(n d-j)=\emptyset .
$$


3.3. Some explicit calculations. One can make explicit calculations by using the antiequivalence between elliptic sheaves and Drinfeld modules ([Dr2], $[\mathrm{Mu}]$ ) and by using the explicit calculation of the global sections $s$, ([Al1, Remark 3.1]), in terms of the $I$-torsion elements of the Drinfeld modules. For $n=1$, calculations are made in [An2] and in the spirit of that work in [Al3, Example 2, p. 21] and in [Al2, 3.2].

We begin with the following example.

EXAMPLE 3.11. We set $n=1, I=p(t) \mathbb{F}_{q}[t], p(t)=\left(t-a_{1}\right) \cdots\left(t-a_{d+1}\right)$ with $a_{i} \neq a_{j}$ for $i \neq j$ and $a_{i} \in \mathbb{F}_{q}$. Let $\left(L_{j}, i_{j}, \tau\right)$ be the rank-1 elliptic sheaf defined over Carlitz's cyclotomic ring $K_{1}^{I \infty}=\mathbb{F}_{q}(\bar{t})[\delta]$, with $\delta$ an element of an algebraic closure of $\mathbb{F}_{q}(t)$ satisfying

$$
\phi_{p(t)}(\delta)=\delta^{q^{d+1}}+\cdots+c_{1} \delta^{q}+p(\bar{t}) \delta=0,
$$

where $\phi$ is the Drinfeld module $\phi_{t}=\tau+\bar{t}$ (Remark 3 of Section 2.2).

Let us consider the $I \infty$-level structure, $\iota_{I \infty}$, for $\left(L_{j}, i_{j}, \tau\right)$. We have

$$
\iota_{I}: L_{0} \rightarrow K_{1}^{I \infty}[t] / p(t)
$$

given by $\iota_{I \infty}(s)=m_{1} \delta_{1} \frac{p(t)}{t-\alpha_{1}}+\cdots+m_{d+1} \delta_{d+1} \frac{p(t)}{t-\alpha_{d+1}}$, and

$$
\iota_{\infty}: L_{0} \rightarrow K_{1}^{I \infty}\left[t^{-1}\right] / t^{-1}
$$

with $\iota_{\infty}(s)=1$. Here $L_{0}=s \cdot \mathcal{O}_{\mathbb{P}^{1}} \otimes K_{1}^{I \infty}, \phi_{p(t) /\left(t-a_{j}\right)}(\delta)=\delta_{j}$ and the $m_{j}$ are obtained from the equality

$$
\frac{1}{p(t)}=\frac{m_{1}}{t-a_{1}}+\cdots+\frac{m_{d+1}}{t-a_{d+1}}
$$

We shall obtain the element of $K_{1}^{I \infty} \otimes K_{1}^{I \infty}$ whose divisor is

$$
T(d)+\Gamma(\mathrm{Fr}) * T(d-1)+\cdots+\Gamma\left(\mathrm{Fr}^{d-1}\right) * T(1)+\Gamma\left(\mathrm{Fr}^{d}\right) .
$$

Let $\pi_{1}$ and $\pi_{2}$ be the natural projections

$$
\operatorname{Spec}\left(K_{1}^{I \infty} \otimes K_{1}^{I \infty}\right) \rightarrow \operatorname{Spec}\left(K_{1}^{I \infty}\right) .
$$

The morphism $h$ of Lemma 3.7 applied to the rank-1 line bundles with an $I$-level structure, $\pi_{1}^{*}\left(L_{0}, \iota_{I}\right)$ and $\pi_{2}^{*}\left(L_{0}(d), \iota_{I}\right)$, is given by

$$
h\left(\pi_{1}^{*} s\right)=\left[m_{1} \frac{\delta_{1} \otimes 1}{1 \otimes \delta_{1}} \frac{p(t)}{t-\alpha_{1}}+\cdots+m_{d+1} \frac{\delta_{d+1} \otimes 1}{1 \otimes \delta_{d+1}} \frac{p(t)}{t-\alpha_{d+1}}\right] \pi_{2}^{*} s .
$$

By Lemma 3.8, one must require that

$$
\pi_{2}^{*} \iota_{\infty}\left(h\left(\pi_{1}^{*} s\right)\right)=\pi_{1}^{*} \iota_{I \infty}\left(\pi_{1}^{*} s\right) .
$$

By the definition of $\infty$-level structures,

$$
\pi_{2}^{*} \iota_{\infty}\left(h\left(\pi_{1}^{*} s\right)\right)=m_{1} \frac{\delta_{1} \otimes 1}{1 \otimes \delta_{1}}+\cdots+m_{d+1} \frac{\delta_{d+1} \otimes 1}{1 \otimes \delta_{d+1}},
$$


which is the leading coefficient of the polynomial

$$
m_{1} \frac{\delta_{1} \otimes 1}{1 \otimes \delta_{1}} \frac{p(t)}{t-a_{1}}+\cdots+m_{d+1} \frac{\delta_{d+1} \otimes 1}{1 \otimes \delta_{d+1}} \frac{p(t)}{t-a_{d+1}} .
$$

Since $\iota_{I \infty}(s)=1$, the element sought is

$$
m_{1} \frac{\delta_{1} \otimes 1}{1 \otimes \delta_{1}}+\cdots+m_{d+1} \frac{\delta_{d+1} \otimes 1}{1 \otimes \delta_{d+1}}-1 .
$$

EXAMPLE 3.12. Now, we consider the easiest non-abelian case with $n=2$, $I=t \mathbb{F}_{q}[t]$. Let $\phi_{t}=a \sigma^{2}+b \sigma+\bar{t}$ be a Drinfeld module of rank two defined over the ring

$$
B_{2}^{I \infty}=\left(\mathbb{F}_{q}\left[a, a^{1 /(1-q)}, b, \bar{t}, r(\bar{t})^{-1}\right] / a+b+\bar{t}-1\right)[\Gamma],
$$

with $\phi_{t}(1)=0, \Gamma^{q}-\Gamma \neq 0$ and $\phi_{t}(\Gamma)=0$. Here $r(t)$ is the product of the monic polynomials of degree less than or equal to 2 . Let $\left(M_{j}, i_{j}, \tau\right)$ be the rank-2 elliptic sheaf associated with $\phi$, and let $\iota_{I \infty}$ be an $I \infty$-level structure for $\left(M_{j}, i_{j}, \tau\right)$ given by

$$
\iota_{I}: M_{0} \rightarrow\left(B_{2}^{I \infty}[t] / t\right)^{\oplus 2} \simeq\left(B_{2}^{I \infty}\right)^{\oplus 2}
$$

with $\iota_{I \infty}(s)=(1, \Gamma)$ and $\iota_{I \infty}(\tau s)=\left(1, \Gamma^{q}\right)$. Recall that

$$
M_{0}=s \cdot\left(\mathcal{O}_{\mathbb{P}^{1}} \otimes B_{2}^{I \infty}\right) \oplus \tau s \cdot\left(\mathcal{O}_{\mathbb{P}^{1}} \otimes B_{2}^{I \infty}\right) .
$$

The $\infty$-level structure

$$
\iota_{\infty}: \operatorname{Det}\left(M_{0}\right) \rightarrow B_{2}^{I \infty}\left[t^{-1}\right] / t^{-1}
$$

is given by $\iota_{\infty}(s \wedge \tau s)=a$.

Let $\pi_{1}$ and $\pi_{2}$ be the natural projections

$$
\operatorname{Spec}\left(B_{2}^{I \infty} \otimes B_{2}^{I \infty}\right) \rightarrow \operatorname{Spec}\left(B_{2}^{I \infty}\right) .
$$

The morphism $h$ of Lemma 3.7 applied to the rank- 2 vector bundles with an $I$-level structure, $\pi_{1}^{*}\left(M_{0}, \iota_{I}\right)$ and $\pi_{2}^{*}\left(M_{0}, \iota_{I}\right)$ (here $d=0$ ), is given by the matrix product

$$
D:=\left(\begin{array}{cc}
1 & 1 \\
1 \otimes \Gamma & 1 \otimes \Gamma^{q}
\end{array}\right)^{-1} \cdot\left(\begin{array}{cc}
1 & 1 \\
\Gamma \otimes 1 & \Gamma^{q} \otimes 1
\end{array}\right) .
$$

By Lemma 3.8, one must require that $\operatorname{deg}\left(h_{A}(1)\right)=0$, where

$$
h_{A}: B_{2}^{I \infty} \otimes B_{2}^{I \infty}\{\tau\} \rightarrow B_{2}^{I \infty} \otimes B_{2}^{I \infty}\{\tau\}
$$

is the restriction of $h$ to $\mathbb{P}^{1} \backslash\{\infty\}$. By considering the second entry of $D\left(\begin{array}{l}1 \\ 0\end{array}\right)$, this condition is

$$
*:=\left(1 \otimes \Gamma^{q}-1 \otimes \Gamma\right)^{-1}(\Gamma \otimes 1-1 \otimes \Gamma)=0 .
$$

We must now impose that

$$
\pi_{2}^{*} \iota_{\infty}\left(\operatorname{det}(h)\left(\pi_{1}^{*}(s \wedge \tau s)\right)\right)=\pi_{1}^{*} \iota_{I \infty}\left(\pi_{1}^{*}(s \wedge \tau s)\right) .
$$


Since $\iota_{\infty}(s \wedge \tau s)=a$, we have

$$
\pi_{2}^{*} \iota_{\infty}\left(\operatorname{det}(h)\left(\pi_{1}^{*}(s \wedge \tau s)\right)\right)=\operatorname{Det}(D) \cdot(1 \otimes a),
$$

and

$$
\pi_{1}^{*} \iota_{I \infty}\left(\pi_{1}^{*}(s \wedge \tau s)\right)=a \otimes 1 .
$$

Thus we obtain the element

$$
* *:=\left(\Gamma^{q}-\Gamma\right) \otimes\left(\Gamma^{q}-\Gamma\right)^{-1}-a \otimes a^{-1} .
$$

The final result is that the diagonal subscheme of $\operatorname{Spec}\left(B_{2}^{I \infty} \otimes B_{2}^{I \infty}\right)$ is the zero locus of the ideal generated by $*$ and $* *$.

4. The additive case: $n=2$ (annihilators for cusp forms of weight 2). In this section, we shall follow the notation set out in the introduction. The set of cusps is $\overline{\mathcal{E}}(I \infty) \backslash \mathcal{E}(I \infty)$. We denote by $\mathcal{C}^{0}(I \infty)$ the divisor class group on $\overline{\mathcal{E}}(I \infty)$ whose support lies among the cusps. As in the introduction we follow the notation and results of [GR]. For the definition and study of cusp forms, the readers are referred to the works of Gekeler, Goss or the Habilitationsschrift of Gebhard Böckle.

We now prove a lemma which is the counterpart for $n=2$ for Stickelberger's theorem. For $n=1$ this is a result of Anderson and Coleman ([An1], [C]).

Lemma 4.1. The correspondence $T(2 d)+T(2 d-1)+\cdots+T(1)+\Gamma(\mathrm{Id})$ annihilates the group $\operatorname{Pic}(\mathcal{E}(I \infty))$.

Proof. This lemma is proved by using Theorem 6.1 below, and the fact that the divisor group, $\mathcal{D}^{0}(\mathcal{E}(I \infty))$, of the affine curve $\mathcal{E}(I \infty)$ defined over $K_{I}^{\infty}$ is a subgroup of the group of Weil divisors of $\mathcal{E}_{2,\left|\mathbb{P}_{1}\right|_{2 d}}^{I \infty}$. Recall that $\mathcal{E}_{2,\left|\mathbb{P}_{1}\right|_{2 d}}^{I \infty}$ is a smooth variety of dimension 2 .

Note that the Hecke correspondences operate on the cusps. Thus, the above correspondence gives a group endomorphism $\mathcal{C}^{0}(I \infty) \rightarrow \mathcal{C}^{0}(I \infty)$. We denote by $\bar{S}_{2}(d), S_{2}(d)$ and $S_{2}^{\prime}(d)$ the group endomorphisms given by

$$
T(2 d)+T(2 d-1)+\cdots+T(1)+\Gamma(\mathrm{Id})
$$

on the groups $\operatorname{Pic}^{0}(\overline{\mathcal{E}}(I \infty)), \operatorname{Pic}(\mathcal{E}(I \infty))$ and $\mathcal{C}^{0}(I \infty)$, respectively.

Let us consider $j^{*}$, the pull back of the line bundles over $\overline{\mathcal{E}}(I \infty)$ by the natural inclusion

$$
j: \mathcal{E}(I \infty) \hookrightarrow \overline{\mathcal{E}}(I \infty) .
$$

We assume that

$$
j^{*}: \operatorname{Pic}^{0}(\overline{\mathcal{E}}(I \infty)) \rightarrow \operatorname{Pic}(\mathcal{E}(I \infty))
$$

is surjective, which is the case, for example, if among the cusp points $\overline{\mathcal{E}}(I \infty) \backslash$ $\mathcal{E}(I \infty)$ there exists a rational point over $K_{I}^{\infty}$. If this does not occur then it suffices to replace $\operatorname{Pic}(\mathcal{E}(I \infty))$ by $\operatorname{Pic}^{0}(\mathcal{E}(I \infty))$. 
Lemma 4.2. If $\operatorname{Pic}(\mathcal{E}(I \infty))$ is an infinite group, then $\operatorname{Ker}\left(\bar{S}_{2}(d)\right)$ is also infinite.

Proof. If $\operatorname{Coker}(S)$ is not finite then the proof is finished, since $\mathcal{C}^{0}(I \infty)$ is a finitely generated group. Thus, we can assume that $\operatorname{Ker}\left(S_{2}^{\prime}(d)\right)$ and $\operatorname{Coker}\left(S_{2}^{\prime}(d)\right)$ are finite groups.

From the serpent lemma applied to the commutative diagram

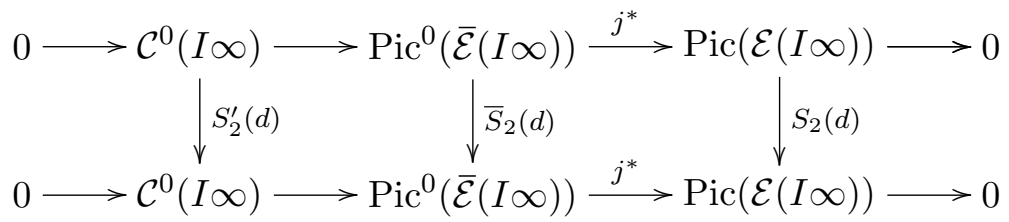

one obtains an exact sequence

$$
\operatorname{Ker}\left(S_{2}^{\prime}(d)\right) \rightarrow \operatorname{Ker}\left(\bar{S}_{2}(d)\right) \rightarrow \operatorname{Pic}(\mathcal{E}(I \infty)) \stackrel{\delta}{\rightarrow} \operatorname{Coker}\left(S_{2}^{\prime}(d)\right) .
$$

This completes the proof since $\operatorname{Ker}\left(S_{2}^{\prime}(d)\right)$ and $\operatorname{Coker}\left(S_{2}^{\prime}(d)\right)$ are finite groups and by hypothesis, $\operatorname{Pic}(\mathcal{E}(I \infty))$ is infinite.

THEOREM 4.3. If the group $\operatorname{Pic}(\mathcal{E}(I \infty))$ is infinite, then there exists a cusp form of weight 2 (and type 1) for $\Gamma_{I \infty}$ that is annihilated by $\widetilde{T}(2 d)+$ $\widetilde{T}(2 d-1)+\cdots+\widetilde{T}(1)+$ Id .

Proof. We denote by $J$ the Jacobian of the curve $\overline{\mathcal{E}}(I \infty)$ over $K_{I}^{\infty}$. Thus, the correspondence $\bar{S}_{2}(d)$ gives an endomorphism of this Jacobian. By the last lemma, this endomorphism cannot be an isogeny. Accordingly, the morphism induced on the tangent space $T_{e}(J)$ of $J$ at the zero element,

$$
\widetilde{S}_{2}(d): T_{e}(J) \rightarrow T_{e}(J),
$$

is not injective. This yields the assertion because $T_{e}(J)$ is the dual of the space of 1-holomorphic differential forms, $H^{0}\left(\overline{\mathcal{E}}(I \infty), \Omega_{\overline{\mathcal{E}}(I \infty) / K_{I}^{\infty}}\right)$, and the space of cusp forms of weight 2 (and type 1 ) is identified with

$$
H^{0}\left(\bar{M}_{\Gamma_{I \infty}}, \Omega_{\bar{M}_{\Gamma_{I \infty}} / C}\right)=H^{0}\left(\overline{\mathcal{E}}(I \infty), \Omega_{\overline{\mathcal{E}}(I \infty) / K_{I}^{\infty}}\right) \otimes_{K_{I}^{\infty}} C .
$$

5. Ideal class group annihilators for cyclotomic function fields. We consider $\mathcal{E}_{1,\left|\mathbb{P}_{1}\right|_{\text {nd }}}^{I \infty}=\operatorname{Spec}\left(\mathcal{B}_{1}^{I \infty}\right)$. The construction of $\mathcal{B}_{1}^{I \infty}$ is detailed in Section 2.2, Remark 3, and is essentially as follows:

Let $\left(\left(L_{j}, i_{j}, \tau\right), \iota_{I \infty}\right)$ be an element of $\mathcal{E}_{1,\left|\mathbb{P}_{1}\right|_{n d}}^{I \infty}$. To construct $\mathcal{B}_{1}^{I \infty}$, we can fix a global section $s$ of $L_{0}$ such that $t \cdot s=\lambda \cdot s+\tau s$ and $\iota_{\infty}(s)=1$. Hence, $\operatorname{Spec}\left(\mathcal{B}_{1}^{I \infty}\right)$ represents the pairs $\left(\phi, \iota_{I}\right)$, with $\phi$ a rank 1-normalized Drinfeld module and $\iota_{I}$ an $I$-level structure for $\phi . \mathcal{B}_{1}^{I \infty}$ is considered in [An1] and [C] and is obtained from the $I$-torsion elements of a normalized Drinfeld module. The "zero" morphism $\mathcal{E}_{1,\left|\mathbb{P}_{1}\right|_{n d}}^{I \infty} \operatorname{Spec}\left(\mathbb{F}_{q}[t]\right)$ gives a Galois 
extension $K_{I}^{\infty} / \mathbb{F}_{q}(t)$ with group $\left(\mathbb{F}_{q}[t] / I\right)^{\times}$. We denote by $Y_{I}^{\infty}$ the proper smooth curve associated with $\mathcal{E}_{1,\left|\mathbb{P}_{1}\right|_{n d}}^{I \infty}$.

We consider the Hecke correspondence

$$
T\left(J_{1}, \ldots, J_{n}\right) \subset \mathcal{E}_{n,\left|\mathbb{P}_{1}\right|_{n d}}^{I \infty} \times \mathcal{E}_{n,\left|\mathbb{P}_{1}\right|_{n d}}^{I \infty},
$$

which is of degree $d\left(J_{1}, \ldots, J_{n}\right)$ over the second component. Let $J$ be the product of ideals

$$
\prod_{i=1}^{n} J_{i}=: J
$$

$T(J)$ denotes the Hecke correspondence on $\mathcal{E}_{1,\left|\mathbb{P}_{1}\right|_{n d}}^{I \infty}$ given by $J$.

There exist actions, $T\left(J_{1}, \ldots, J_{n}\right)^{*}$ and $T(J)^{*}$, of these correspondences on the functors $\underline{\operatorname{Pic}}\left(\mathcal{E}_{n,\left|\mathbb{P}_{1}\right|_{n d}}^{I \infty}\right)$ and $\underline{\operatorname{Pic}}^{0}\left(Y_{I}^{\infty}\right)$, respectively. These functors are defined over the category of $\mathbb{F}_{q}$-schemes. Recall that the projections

$$
\bar{\pi}_{1}, \bar{\pi}_{2}: T\left(J_{1}, \ldots, J_{n}\right) \rightarrow \mathcal{E}_{n,\left|\mathbb{P}_{1}\right|_{n d}}^{I \infty}
$$

are étale. In this way it is possible to define $T\left(J_{1}, \ldots, J_{n}\right)^{*}:=\left(\bar{\pi}_{2}\right)_{*} \cdot \bar{\pi}_{1}^{*}$.

REMARK 4. Consider the morphism det $: \mathcal{E}_{n,\left|\mathbb{P}_{1}\right|_{n d}}^{I \infty} \rightarrow \mathcal{E}_{1,\left|\mathbb{P}_{1}\right|_{n d}}^{I \infty}$. Then

$$
T\left(J_{1}, \ldots, J_{n}\right)^{*} \operatorname{det}^{*}[D]=d\left(J_{1}, \ldots, J_{n}\right) \operatorname{det}^{*} T(J)^{*}[D],
$$

where $[D]$ is the class of a divisor $D$ on $\mathcal{E}_{1,\left|\mathbb{P}_{1}\right|_{n d}}^{I \infty}$.

The above equality is proved bearing in mind the projection formula for the rational equivalence of cycles; that $\bar{\pi}_{2}$ is an étale morphism of degree $d\left(J_{1}, \ldots, J_{n}\right)$; and that given $e:=\left(E, \iota_{I \infty}\right) \in \mathcal{E}_{n}^{I \infty}$ we have

$$
\begin{gathered}
\bar{\pi}_{2}\left[T\left(J_{1}, \ldots, J_{n}\right) \cap\left(\operatorname{det}^{-1}(\operatorname{det}(e)) \times \mathcal{E}_{n,\left|\mathbb{P}_{1}\right|_{n d}}^{I \infty}\right)\right]=\operatorname{det}^{-1}\left(T(J)^{*} \operatorname{det}(e)\right), \\
T\left(J_{1}, \ldots, J_{n}\right) \cap\left(\operatorname{det}^{-1}(\operatorname{det}(e)) \times \mathcal{E}_{n,\left|\mathbb{P}_{1}\right|_{n d}}^{I \infty}\right)=\bar{\pi}_{2}^{-1}\left[\operatorname{det}^{-1}\left(T(J)^{*} \operatorname{det}(e)\right)\right] .
\end{gathered}
$$

Lemma 5.1. The correspondence

$$
D_{I}^{n}:=\sum_{i=0}^{n d-i} \Gamma\left(\mathrm{Fr}^{i}\right) *\left[\sum_{\substack{J \subset \mathbb{F}_{q}[t] \\ J+I=\mathbb{F}_{q}[t], \operatorname{deg}(J)=i}}\left(\sum_{\substack{J_{1} \subseteq \cdots \subseteq J_{n} \\ \prod_{k=1}^{n}=J_{k}=J}} d\left(J_{1}, \ldots, J_{n}\right)\right) T(J)\right]
$$

is trivial on $Y_{I}^{\infty} \times Y_{I}^{\infty}$ up to vertical and horizontal correspondences.

Proof. It suffices to consider a curve $Z \rightarrow \mathcal{E}_{n,\left|\mathbb{P}_{1}\right|_{n d}}^{I \infty}$ such that the morphism composition

$$
g: Z \rightarrow \mathcal{E}_{n,\left|\mathbb{P}_{1}\right|_{n d}}^{I \infty} \stackrel{\text { det }}{\longrightarrow} \mathcal{E}_{1,\left|\mathbb{P}_{1}\right|_{n d}}^{I \infty}
$$

is not constant. By the above remark, $(\operatorname{det})^{*} \cdot\left(D_{I}^{n}\right)^{*}=\left(T_{I}^{n}\right)^{*} \cdot(\operatorname{det})^{*}$. Since $T_{I}^{n}$ is rationally equivalent to zero, $(g)_{*}(g)^{*} \cdot\left(D_{I}^{n}\right)^{*}$ is trivial on $Y_{I}^{\infty} \times Y_{I}^{\infty}$ up 
to vertical and horizontal correspondences, but by the projection formula,

$$
(g)_{*}(g)^{*} \cdot\left(D_{I}^{n}\right)^{*}=m \cdot D_{I}^{n}
$$

with some $m \in \mathbb{N}$. This yields the assertion, since the ring of correspondences, modulo horizontal and vertical ones, is without $\mathbb{Z}$-torsion.

We consider $J=q(t) \mathbb{F}_{q}[t]$ with $q(t)$ monic. Then $T(J)$ is given by the graph, $\Gamma(q(t))$ of the automorphism of $\mathcal{E}_{1,\left|\mathbb{P}_{1}\right|_{\text {nd }}}^{I \infty}$ obtained from the action of $q(t) \in\left(\mathbb{F}_{q}[t] / I\right)^{\times}$. Recall that to obtain $\mathcal{B}_{1}^{I \infty}$ we have fixed a global section $s$ of $L_{0}$ such that $t \cdot s=\lambda \cdot s+\tau s$, and $\iota_{\infty}(s)=1$. In this way, $T(J)=\Gamma(q(t))$. By Section 2.3, if we set $J_{i}=q_{i}(t) \mathbb{F}_{q}[t]$, then

$$
\varphi(q(t), n):=\sum_{\substack{J_{1} \subseteq \cdots \subseteq J_{n} \\ \prod_{k=1}^{n} \subseteq J_{k}=J}} d\left(J_{1}, \ldots, J_{n}\right)
$$

is the number of submodules $N \subseteq \mathbb{F}_{q}[t]^{\oplus n}$ such that

$$
\mathbb{F}_{q}[t]^{\oplus n} / N \simeq \mathbb{F}_{q}[t] / q_{1}(t) \oplus \cdots \oplus \mathbb{F}_{q}[t] / q_{n}(t),
$$

with the product of the invariant factors $q_{1}(t) \cdots q_{r}(t)$ equal to $q(t)$. Therefore, if we consider $p(t) \mathbb{F}_{q}[t]=I$, then the following corollary can be deduced from the Euler product of Theorem 2.7 and Anderson and Coleman's results ([An1], [C]).

Corollary 5.2. The correspondence

$$
\sum_{i=0}^{n d}\left[\Gamma\left(\operatorname{Fr}^{n d-i}\right) *\left(\sum_{\substack{m o n i c \\(p(t), q(t)) \in \mathbb{F}_{q}[t]}} \varphi(q(t), n) \cdot \Gamma(q(t))\right)\right]
$$

is trivial on $Y_{I}^{\infty} \times Y_{I}^{\infty}$ up to vertical and horizontal correspondences.

EXAMPLE 5.3. We can check this result for $n=2$ and $p(t)=t(t-1)$. Let $K_{t(t-1)}^{\infty} / \mathbb{F}_{q}(t)$ be the Galois extension of group $\left(\mathbb{F}_{q}[t] / t(t-1)\right)^{\times}$.

One has

$$
\begin{aligned}
\varphi(t-\alpha, 2) & =q+1, & \varphi((t-\alpha)(t-\beta), 2) & =q^{2}+2 q+1, \\
\varphi\left((t-\alpha)^{2}, 2\right) & =q^{2}+q+1, & \varphi\left(t^{2}+a t+b, 2\right) & =q^{2}+1
\end{aligned}
$$

with $t^{2}+a t+b \in \mathbb{F}_{q}[t]$ an irreducible polynomial and $\alpha, \beta \in \mathbb{F}_{q}, \alpha \neq \beta$. Thus

$$
\sum_{i=0}^{2}\left[\Gamma\left(\mathrm{Fr}^{2-i}\right) *\left(\sum_{\substack{\operatorname{monic} q(t) \in \mathbb{F}_{q}[t] \\(t(t-1), q(t))=1, \operatorname{deg}(q(t))=i}} \varphi(q(t), 2) \cdot \Gamma(q(t))\right)\right]
$$


is

$$
\begin{aligned}
\Gamma\left(\mathrm{Fr}^{2}\right) & +(q+1) \sum_{\alpha \neq 0,1} \Gamma(\mathrm{Fr}) * \Gamma(t-\alpha) \\
& +\left(q^{2}+2 q+1\right) \sum_{\substack{\{\alpha, \beta\} \subset \mathbb{F}_{q} \\
\alpha, \beta \neq 0,1 \\
\alpha \neq \beta}} \Gamma((t-\alpha)(t-\beta)) \\
& +\left(q^{2}+q+1\right) \sum_{\substack{\alpha \neq 0,1\\
}} \Gamma\left((t-\alpha)^{2}\right) \\
& +\left(q^{2}+1\right) \sum_{\substack{a, b \in \mathbb{F}_{q} \\
t^{2}+a t+b \text { irreducible }}} \Gamma\left(t^{2}+a t+b\right),
\end{aligned}
$$

and grouping terms, we have

$$
\begin{aligned}
& \Gamma(\mathrm{Fr}) *\left[\Gamma(\mathrm{Fr})+\sum_{\alpha \neq 0,1} \Gamma(t-\alpha)\right] \\
& +q\left[\sum_{\alpha \neq 0,1} \Gamma(t-\alpha)\right] *\left[\Gamma(\mathrm{Fr})+\sum_{\alpha \neq 0,1} \Gamma(t-\alpha)\right] \\
& +\left(q^{2}+1\right)\left[\sum_{\substack{\{\alpha, \beta\} \subset \mathbb{F}_{q} \\
\alpha, \beta \neq 0,1 \\
\alpha \neq \beta}} \Gamma((t-\alpha)(t-\beta))+\sum_{\alpha \neq 0,1} \Gamma\left((t-\alpha)^{2}\right)+\sum \Gamma\left(t^{2}+a t+b\right)\right] .
\end{aligned}
$$

Now, bearing in mind that the last summand is

$$
\left(q^{2}+1\right) \sum_{g \in\left(\mathbb{F}_{q}[t] / t(t-1)\right)^{\times}} \Gamma(g),
$$

which is a trivial correspondence, we conclude that $(*)$ is also trivial because the correspondence

$$
\Gamma(\mathrm{Fr})+\sum_{\alpha \in \mathbb{F}_{q} \backslash\{0,1\}} \Gamma(t-\alpha)
$$

is trivial on $K_{t(t-1)}^{\infty} \otimes K_{t(t-1)}^{\infty}($ see $[\mathrm{C}])$.

6. The above results without $\infty$-level structures. With minor changes in the above results one can obtain similar results but over the modular varieties $\mathcal{E}_{n}^{I}$. The results obtained match, for $n=1$, the classical Stickelberger theorem over $\mathbb{Z}$ (see [An1], [C], [Gr1] and [Gr2]).

To obtain these results it suffices to replace in Lemma 3.8 the condition imposed on $h$ to be a morphism of $\infty$-level structures, by the condition

$$
\operatorname{deg}_{\bar{\tau}}\left(h_{A}\left(\tau^{n}\right)-\bar{\tau} \cdot h_{A}\left(\tau^{n-1}\right)\right) \leq n-1+n d
$$


And now in Corollary 3.5 one allows pairs, $\left[\left(E, \iota_{I}\right),\left(\bar{E}, \bar{\iota}_{I}\right)\right]$, given by an isogeny for $I$-level structures, $\Phi:\left(E, \iota_{I}\right) \rightarrow\left(\bar{E}, \bar{\iota}_{I}\right)$, such that $\infty$ can be in $\operatorname{supp}(\bar{E} / \Phi(E))$. Thus, one obtains:

TheOREM 6.1. The correspondence

$$
\begin{array}{r}
T(n d)+[\Gamma(\mathrm{Fr})+\Gamma(\mathrm{Id})] * T(n d-1)+\cdots+\left[\Gamma\left(\mathrm{Fr}^{n d-1}\right)+\cdots+\Gamma(\mathrm{Id})\right] * T(1) \\
+\left[\Gamma\left(\mathrm{Fr}^{n d}\right)+\Gamma\left(\mathrm{Fr}^{n d-1}\right)+\cdots+\Gamma(\mathrm{Fr})+\Gamma(\mathrm{Id})\right]
\end{array}
$$

is trivial (= rationally equivalent to 0 as an $n$-cycle) in $\mathcal{E}_{n,\left|\mathbb{P}_{1}\right|_{n d}}^{I} \times \mathcal{E}_{n,\left|\mathbb{P}_{1}\right|_{n d}}^{I}$.

From the last theorem one has, for $n=2$ :

Lemma 6.2. The correspondence

$$
T(2 d)+2 T(2 d-1)+\cdots+2 d T(1)+(2 d+1) \Gamma(\mathrm{Id})
$$

annihilates the group $\operatorname{Pic}(\mathcal{E}(I))$.

THEOREM 6.3. If the group $\operatorname{Pic}(\mathcal{E}(I))$ is infinite, then there exists a cusp form of weight 2 (and type 1) for $\Gamma_{I}$ that is annihilated by

$$
\widetilde{T}(2 d)+2 \widetilde{T}(2 d-1)+\cdots+2 d \widetilde{T}(1)+(2 d+1) \Gamma(\mathrm{Id}) .
$$

Acknowledgments. I would like to thank the referee for suggesting to deal with annihilators for cusp forms. I would like to thank N. Skinner for doing his best to supervise my deficient English. I am also deeply grateful to Ricardo Alonso Blanco and Jesús Muñoz Díaz for their help.

\section{References}

[Al1] A. Álvarez, Uniformizers for elliptic sheaves, Int. J. Math. 11 (2000), 949-968.

[Al2] -, The theta divisor and the Stickelberger theorem, Proc. Amer. Math. Soc. 133 (2005), 2207-2217.

[Al3] - Zeta correspondences in rank-n, arxiv:mat/0211207.

[An1] G. Anderson, A two dimensional analogue of Stickelberger's theorem, in: The Arithmetic of Function Fields, D. Goss and D. R. Hayes (eds.), de Gruyter, Berlin, 1992, 51-77.

[An2] -, Rank one elliptic A-modules and A-harmonic series, Duke Math. J. 73 (1994), 491-542.

[BlSt] A. Blum and U. Stuhler, Drinfeld modules and elliptic sheaves, in: Vector Bundles on Curves-New Directions, S. Kumar et al. (eds.), Lecture Notes in Math. 1649, Springer, 1997, 110-188.

[Ca] L. Carlitz, On certain functions connected with polynomials in a Galois field, Duke Math. J. 1 (1935), 137-168.

[C] R. Coleman, On the Frobenius endomorphisms of the Fermat and Artin-Schreier curves, Proc. Amer. Math. Soc. 102 (1988), 463-466.

[Dr1] V. G. Drinfeld, Elliptic modules, Math. USSR-Sb. 23 (1976), 561-592.

[Dr2] - Commutative subrings of certain non-commutative rings, Funct. Anal. Appl. 11 (1977), 9-12. 
[Dr3] V. G. Drinfeld, Varieties of modules of F-sheaves, ibid. 21 (1987), 107-122.

[GR] E. U. Gekeler and M. Reversat, Jacobians of Drinfeld modular curves, J. Reine Angew. Math. 476 (1996), 27-93.

[Ge] A. Genestier, Espaces symétriques de Drinfeld, Astérisque 234 (1996).

[Gr1] B. Gross, The annihilation of divisor classes in abelian extensions of the rational function field, in: Séminaire de Théorie des Nombres, Bordeaux, 1980-1981, exp. 3,5 pp.

[Gr2] - Algebraic Hecke characters for function fields, in: Séminaire de Théorie des Nombres, Paris, 1980-1981, Progr. Math. 22, Birkhäuser, 1982, 87-90.

[H1] D. Hayes, Stickelberger elements in function fields, Compos. Math. 55 (1993), 251-292.

[H2] - Stickelberger functions for non-abelian Galois extensions of global fields, in: Stark's Conjectures: Recent Work and New Directions, Contemp. Math. 358, Amer. Math. Soc., Providence, RI, 2004, 193-206.

[Lf] L. Lafforgue, Chtoucas de Drinfeld et conjecture de Ramanujan-Peterson, Astérisque 243 (1997).

[Ln] S. Lang, Introduction to Modular Forms, I, Springer, Berlin, 1976.

[Lm] G. Laumon, Cohomology of Drinfeld Modular Varieties I, Cambridge Univ. Press, 1996.

[LRSt] G. Laumon, M. Rapoport and U. Stuhler, D-elliptic sheaves and the Langlands correspondence, Invent. Math. 113 (1993), 217-338.

[Ma] P. A. Macmahon, Combinatorial Analysis, Chelsea, 1960.

$[\mathrm{Mu}] \quad$ D. Mumford, An algebro-geometric construction of commuting operators and of solutions to the Toda lattice equations, Korteweg-de Vries equation and related non-linear equations, in: Int. Symp. Algebraic Geometry (Kyoto, 1977), Kinokuniya, Tokyo, 1977, 115-153.

[Sh] G. Shimura, Introduction to the Arithmetic Theory of Automorphic Functions, Publ. Math. Soc. Japan, Tokyo-Princeton, 1971.

[Ta] J. Tate, Les conjectures de Stark sur les fonctions L d'Artin en $s=0$, Birkhäuser, Boston, 1984.

Departamento de Matemáticas

Universidad de Salamanca

Plaza de la Merced 1-4

37008 Salamanca, Spain

E-mail: aalvarez@usal.es

Received on 15.12.2006

and in revised form on 4.9.2008 